\title{
Worldwide Variations in Demographics, Management, and Outcomes of Acute Pancreatitis
}

\author{
Bassem Matta ${ }^{1}$, Amir Gougol $^{2},{\text { Xiaotian } \mathrm{Gao}^{3} \text {, Nageshwar Reddy }}^{4}$, Rupiyoti Talukdar ${ }^{5}$, Rakesh Kochhar ${ }^{6}$, \\ Mahesh Kumar Goenka ${ }^{7}$, Aiste Gulla ${ }^{8}$, Jose A. Gonzalez ${ }^{9}$, Vikesh K. Singh ${ }^{10}$, Miguel Ferreira ${ }^{11}$, Tyler \\ Stevens $^{12}$, Sorin T. Barbu ${ }^{13}$, Haq Nawaz ${ }^{14}$, Silvia C. Gutierrez ${ }^{15}$, Narcis O. Zarnescu ${ }^{16}$, Gabriele \\ Capurso $^{17}$, Jeffrey Easler ${ }^{18}$, Konstantinos Triantafyllou ${ }^{19}$, Mario Pelaez-Luna ${ }^{20}$, Shyam Thakkar $^{21}$, Carlos \\ Ocampo $^{22}$, Enrique de-Madaria ${ }^{23}$, Gregory A. Cote ${ }^{24}$, Bechien U. Wu ${ }^{25}$, Pedram Paragomi ${ }^{26}$, Ioannis \\ Pothoulakis $^{27}$, Gong Tang ${ }^{28}$, \& Georgios I. Papachristou ${ }^{29,30}$ \\ 1, Bassem Matta: matta.bassem@gmail.com \\ University of Pittsburgh Medical Center, Pittsburgh, Pennsylvania, USA
}

2, Amir Gougol: gougolah@upmc.edu

University of Pittsburgh Medical Center, Pittsburgh, Pennsylvania, USA

1 and 2 contributed equally as first authors

3, Xiaotian Gao: XIG31@ pitt.edu

Department of Biostatistics, School of Public Health, University of Pittsburgh, Pittsburgh, Pennsylvania, USA

4. Nageshwar Reddy: aigindia@yahoo.co.in

Asian Gastroenterology Institute, Hyderabad, India

5, Rupiyoti Talukdar: rup_talukdar@yahoo.com

Asian Gastroenterology Institute, Hyderabad, India

6, Rakesh Kochhar: dr_kochhar@hotmail.com

Postgraduate Institute of Medical Education and Research, Chandigarh, India

7, Mahesh Kumar Goenka: mkgkolkata@gmail.com

Apollo Gleneagles Hospitals Kolkata, Kolkata, India

8 Aiste Gulla: agulla1@jhu.edu

Lithuanian University of Health Sciences, Kaunas, Lithuania

This is the author's manuscript of the article published in final edited form as:

Matta, B., Gougol, A., Gao, X., Reddy, N., Talukdar, R., Kochhar, R., ... Papachristou, G. I. (2019). Worldwide Variations in Demographics, Management, and Outcomes of Acute Pancreatitis. Clinical Gastroenterology and Hepatology. 
9, Jose A. Gonzalez: joseagonz@yahoo.com

Universidad Autónoma de Nueva León, Monterrey, Mexico

10, Vikesh Singh, vsingh1@jhmi.edu

Johns Hopkins Medical Institutions, Baltimore, Maryland, USA

11, Miguel Ferreira: migue.ferbo@hotmail.com

Hospital Nacional de Itauguá, Itaugua, Paraguay

12, Tyler Stevens: STEVENT@ccf.org

Cleveland Clinic Foundation, Cleveland, Ohio, USA

13, Sorin T. Barbu: barbu@ pancreasclub.ro

University of Medicine and Pharmacy "Iuliu Hatieganu", Cluj-Napoca, Romania;

14, Haq Nawaz: hnawaz@emhs.org

Eastern Maine Medical Center, Maine, Bangor, USA

15, Silvia C. Gutierrez: silviac.gutierrez@gmail.com

Hospital Nacional "Profesor Alejandro Posadas", Buenos Aires, Argentina

16, Narcis O. Zarnescu: nzarnescu@gmail.com

University of Medicine and Pharmacy, Bucharest, Romania

17, Gabriele Capurso: capurso.gabriele@ @sr.it

San Raffaele Scientific Institute, Vita Salute San Raffaele University, Milan, Italy

18, Jefferey Easler: jjeasler@iu.edu

Indiana University School of Medicine, Indianapolis, Indiana, USA

19, Konstantinos Triantafyllou: chronisgatos@gmail.com

Attikon University General Hospital, Athens, Greece 
20, Mario Pelaez-Luna: mariopl@ prodigy.net.mx

Instituto Nacional de Ciencias Médicas y Nutrición Salvador Zubirán-Universidad Autónoma de Mexico, Mexico City, Mexico

21, Shyam Thakhar: sthakkar@wpahs.org

Allegheny General Hospital, Pittsburgh, Pennsylvania, USA

22, Carlos Ocampo: ocampoc@yahoo.com

Hospital General de Argudos "Dr. Cosme Argerich", Buenos Aires, Argentina

23, Enrique De-Madaria: madaria@ hotmail.com

Investigación Sanitaria y Biomédica de Alicante (ISABIAL - Fundación FISABIO), Alicante, Spain

24, Gregory A. Cote: cotea@musc.edu

Medical University of South Carolina, Charleston, South Carolina, USA

25, Bechien Wu: Bechien.U.Wu@kp.org

Kaiser Permanente, Pasadena, California, USA

26, Pedram Paragomi: paragomi@ @itt.edu

University of Pittsburgh Medical Center, Pittsburgh, Pennsylvania, USA

27, Ioannis Pothoulakis, gianhspoth1@gmail.com

University of Pittsburgh Medical Center, Pittsburgh, Pennsylvania, USA

28, Gong Tang, got1@ @itt.edu

Department of Biostatistics, School of Public Health, University of Pittsburgh, Pittsburgh, Pennsylvania, USA

29, 30 Georgios Papachristou: papachri@ pitt.edu

University of Pittsburgh Medical Center, Pittsburgh, Pennsylvania, USA

Ohio State University Wexner Medical Center, Columbus, Ohio, USA 
Short Title: Geographic Variations in Acute Pancreatitis

Corresponding author: Georgios Papachristou, MD, PhD

Title: Professor of Medicine at the Ohio State University Wexner Medical Center, Columbus, $\mathrm{OH}$.

Email: georgios.papachristou@osumc.edu

Telephone number: +1 (614) 293-6255

Fax number: +1 (614) 293-8518

Office: $410 \mathrm{~W} 10^{\text {th }}$ Avenue, $2^{\text {nd }}$ floor, Columbus $\mathrm{OH}, 43210$

None of the authors have published, posted, or submitted any related papers from the same study. 


\section{Acknowledgments:}

- Ayesha Kamal, M.D.

- Benjamin Click, M.D.

- David Whitcomb, M.D., PhD.

- Dhiraj Yadav, M.D., MPH.

- Efstratios Koutroumpakis, M.D.

- Peter Jun Woo Lee, MBChB.

- Phil J Greer, M.S.

- Venkata, Akhintala, M.D.

\section{Author contributions:}

- Statistical analysis: Xiaotian Gao and Gong Tang.

- Drafting of the manuscript: Bassem Matta, Amir Gougol, and Georgios I. Papachristou.

- Data collection, data interpretation, review of manuscript for important intellectual content, final approval of the manuscript: all authors

\section{Conflict of interest and financial disclosures:}

None of the authors have any conflict of interest or pertinent financial disclosures. 


\section{Abbreviations:}

AGA: American gastroenterological association

AP: acute pancreatitis

APPRENTICE: acute pancreatitis patient registry to examine novel therapies in clinical experience

BMI: body mass index

CI: confidence interval

DUA: data use agreements

ERCP: endoscopic retrograde cholangiopancreatography

ICU: intensive care unit

IQR: interquartile range

IRB: institutional review board

RAC: revised Atlanta classification

REDCap: Research Electronic Data Capture

LOS: length of stay

SIRS: systemic inflammatory response syndrome

TPN: total parenteral nutrition 


\section{Abstract:}

Background \& Aims: Few studies have compared regional differences in acute pancreatitis. We analyzed data from an international registry of patients with acute pancreatitis to evaluate geographic variations in patient characteristics, management, and outcomes.

Methods: We collected data from the APPRENTICE registry of patients with acute pancreatitis, which obtains information from patients in Europe (6 centers), India (3 centers), Latin America (5 centers), and North America (8 centers) using standardized questionnaires. Our final analysis included 1,612 patients with acute pancreatitis (median age, 49 years; $53 \%$ male, $62 \%$ white) enrolled from August 2015 through January 2018.

Results: Biliary (45\%) and alcoholic acute pancreatitis (21\%) were the most common etiologies. Based on the revised Atlanta classification, $65 \%$ of patients developed mild disease, $23 \%$ moderate, and $12 \%$ severe. The mean age of patients in Europe (58 years) was older than mean age for all 4 regions (46 years) and a higher proportion of patients in Europe had comorbid conditions ( $73 \%$ vs $50 \%$ overall). The predominant etiology of acute pancreatitis in Latin America was biliary (78\%), whereas alcohol-associated pancreatitis accounted for the highest proportion of acute pancreatitis cases in India (45\%). Pain was managed with opioid analgesics in $93 \%$ of patients in North America versus $27 \%$ of patients in the other 3 regions.

Cholecystectomies were performed at the time of hospital admission for most patients in Latin America (60\% vs $15 \%$ overall). A higher proportion of European patients with severe acute pancreatitis died during the original hospital stay (44\%) compared with the other 3 regions $(15 \%)$ 
Conclusions: We found significant variation in demographics, etiologies, management practices, and outcomes of acute pancreatitis worldwide.

ClinicalTrials.gov number: NCT03075618

KEY WORDS: pancreas; inflammation, drug, treatment 


\section{Introduction}

Acute pancreatitis (AP) is a global leading cause of gastrointestinal-related hospital admissions ${ }^{1}$. The incidence of AP has been reported to be increasing in the United States and Europe ${ }^{2,3}$. Approximately $20 \%$ of people affected develop severe disease resulting in relatively high morbidity and mortality ${ }^{4}$. Over the last decade, multiple advances have occurred in management of AP such as the development of the revised Atlanta classification of disease severity (RAC), introduction of early goal-directed intravenous fluid resuscitation, and implementation of a minimally invasive step-up approach in subjects with symptomatic necrotic pancreatic collections ${ }^{5-7}$. Possibly as a consequence of these developments, case fatality of AP may have decreased however, estimates tend to vary among different countries ${ }^{8,9}$.

Large, multicenter studies in AP from national registries have been recently published. However, these have been confined to national bounds, with the majority being in North America and Europe $^{10-13}$. Results from these studies have revealed heterogeneity in patient characteristics such as demographics, etiology, and risk factors of severe disease. For instance, a large Spanish study from 2018 revealed an AP mortality rate of $4.2 \%$ compared to $1 \%$ from recent reports in the United States ${ }^{13,14}$. Inconsistent severity definitions and methodology hinder the combination and comparison of data from different regions. Furthermore, it is unclear whether recent advances in management of AP have gained traction throughout different areas of the world.

Lack of prospective, multi-national data in AP prompted investigators around the world to create a multi-center collaboration referred as Acute Pancreatitis Patient Registry to Examine Novel Therapies in Clinical Experience (APPRENTICE) ${ }^{15}$. This study's aim was to evaluate the geographic differences in patient characteristics, management, and outcomes of AP across four different geographic areas using APPRENTICE data. 


\section{Methods}

\section{Study Population}

APPRENTICE is a prospective, multicenter, international consortium studying clinical characteristics of AP patients across the world ${ }^{15}$. The University of Pittsburgh served as the coordinating center. Ethical committee approvals were obtained from local institutional review boards (IRB) at all participating centers. University of Pittsburgh's IRB approved this study and acted as an umbrella IRB for incoming centers (PRO15040389). The study was registered in clinicaltrials.gov (NCT03075618). Details on design and methodology of APPRENTICE have been previously published ${ }^{15}$. Adults ( $\geq 18$ years old) admitted with the diagnosis of AP, willing to participate in the study, and enrolled within 2 weeks of presentation were eligible for inclusion. Patients with a history of organ transplantation, trauma induced AP, chronic pancreatitis, and pancreatic cancer were excluded. Enrollment occurred between October 2015 and January 2018. Site investigators were responsible for identifying eligible hospital admitted patients through different screening mechanisms. In total, data from 22 sites, which reached a set minimum number of enrollment $(\geq 15$ patients/center), were included for statistical analysis (Table 1 , Figure 1).

\section{Data collection}

Study questionnaires were carefully designed by recognized experts in the field (appendix table 1). A well-established, secure, web-based, electronic data collection software (Research Electronic Data Capture, REDCap) was used ${ }^{16}$. A test period of 3 months was initially undertaken with the goal to assess applicability and quality of the questionnaires. Multiple online sessions with study personnel (site investigators, coordinators) were conducted prior to, and 
during the enrollment phase in order to ensure the uniformity of data collection, answer questions, and address technical issues. De-identified data was collected prospectively at different hospitalization time points: admission, day 1 , day 2 , day 3 , day 7 , and discharge. Data quality was routinely monitored by a dedicated statistician at the coordinating site. Definition of different collected variables are outlined in appendix table 2.

The primary clinical outcomes of interest included RAC severity, LOS, and in-hospital mortality. Additional outcomes included AP etiology, fluid volume in the first 24 hours of admission, fluid type, analgesic use, feeding methods, and ERCP, or cholecystectomy rates in cases of biliary pancreatitis. All authors had access to the study data and reviewed and approved the final manuscript.

\section{Statistical analysis}

Statistical analysis was performed by expert biostatisticians (X.G., G.T.) at the coordinating center. Continuous variables were summarized by median and interquartile range (IQR).

Categorical variables were presented with proportions of study subjects. Preliminary comparisons of outcome variables among various geographic areas, were performed using the Fisher's exact test for categorical values, and the nonparametric Kruskal-Wallis test was used for continuous variables (Tables 2-5). These were used as global tests that compared patient characteristic and clinical outcomes of interest through all four regions. Significance was defined as a p-value equal to or less than 0.05 ; no adjustment for multiple testing was made in these exploratory analyses.

Subsequently, we focused on the primary clinical outcomes and multivariate regression models were applied to assess whether LOS, severity, and mortality differ among the four geographic 
areas, adjusting for other patient characteristics. The geographic regions were coded by three dummy variables, with North America as the reference region. For multivariable analysis, a linear regression was used to evaluate LOS differences among geographic areas, and logistic regression was used to assess differences in severity (severe AP vs. others) and mortality (severe patients) among different regions. Such differences in outcomes between a region (Europe, India, or Latin America) and North America were presented as odds ratios in the case of severity and mortality, or as associated model coefficients in the case of LOS (Appendix Tables 3-5).

Multivariable models were run including the following covariates: age, gender, body mass index (BMI), Charlson Comorbidity Index, etiology, transfer status, cholecystectomy during the same admission, narcotic use, and severity (only for LOS). The covariates of age, BMI, Charlson Comorbidity Index, and etiology were constantly kept in the model for more accurate prediction, while the remaining covariates dropped when not significant. The likelihood ratio test was used to compare the nested model with region and the adjusted variables as covariates and the submodel with only the adjusted variables as covariates. All analyses were performed in R (Version

\subsection{1, R Foundation).}

\section{Study participants:}

In total, 1,680 AP patients were enrolled between August 2015 and January 2018; 68 were omitted from the analysis yielding a final number of 1,612 subjects. Exclusion of the above subjects was related to removal of sites with $<15$ subjects enrolled from the analysis (13 patients), as part of the predetermined study criteria, or due to missing RAC data (55 patients; Table 1, Figure 1).

\section{Results}




\section{Baseline Characteristics and Etiology}

Out of the 1,612 patients, median age was 49 (IQR, 34-64), and 47\% were females. Biliary (45\%) and alcoholic (21\%) were the most common pancreatitis etiologies (Table 2). Based on RAC, $65 \%$ were classified as developing mild disease, $23 \%$ as moderately severe, and $12 \%$ as severe disease. Median LOS was 8 days (IQR, 5-13, Table 4). Overall, 45 patients died (2.8\%) during their hospitalization (Table 5).

Age, gender, ethnicity, and race distributions differed significantly by geographic areas. Patients from Indian sites were mostly males (75\%), younger in age (39 years, IQR: 30-50) with alcohol being the predominant etiology ( $45 \%$ vs. $14 \%$ in remaining geographic areas, p <0.001). Latin American patients were mostly young (median age 43, IQR 29-59), females (67\%) with the majority of AP linked to biliary etiology ( $78 \%$ vs. $37 \%, \mathrm{p}<0.001)$. In contrast, European and North American subjects had a relatively equal gender distribution, with an overall older age [58, (IQR 45-74) and 52 (IQR 37-65) respectively, $\mathrm{p}<0.001$ ]. Post ERCP pancreatitis was significantly more common in North American sites (19\% vs $2.8 \%$ in remaining geographic areas, $\mathrm{p}<0.001$ ) (Table 2). These differences were mostly driven by two North American sites with 50 out of 90 and 22 out of 62 enrolled patients classified as post ERCP pancreatitis, respectively.

\section{Management}

Data on patient management is presented in table 3. The amount of intravenous fluids administered over the first 24 hours was relatively similar between India, Latin America and North America (ranged between 3-3.2 liters); however, was significantly lower in Europe (2.5 liters, $\mathrm{p}<0.001)$. Lactated Ringers $(\mathrm{LR})$ and normal saline were the two main types of 
intravenous fluids administered in all regions except Latin America. LR was the dominant type of fluid in India (92\%) in contrast to Latin America, where it was rarely used (7\%, p <0.001). The major types of fluids given in Latin America were normal saline (61\%) and Hartman's (32\%); a balanced solution similar to LR, which is not widely available in this region.

The utilization of analgesics was markedly variable across the world. In Europe, non-steroidal anti-inflammatory medications (NSAIDs) comprised the mainstay of pain management (68\%). Indian sites, however, used tramadol in $91 \%$ of their patients, while Latin American centers frequently used opioids (59\%), NSAIDs (48\%), and tramadol (34\%). In contrast, opioid analgesics constituted the cornerstone of analgesia in North America at $93 \%$ of subjects in contrast to $27 \%$ in the remaining regions $(\mathrm{p}<0.001)$. Furthermore, $64 \%$ of subjects in North America were discharged on opioid analgesics compared to $2.7 \%$ in other geographic areas $(\mathrm{p}<0.001)$

European centers had the highest ratio of enteral to parenteral nutrition at $10: 1$ (32\% vs. $3 \%$ in subjects with moderate or severe disease); whereas, total parenteral nutrition (TPN) was most commonly administered in India in $27 \%$ of patients compared to $20 \%$ receiving enteral nutrition (ratio <1:1). The frequency of ERCP among subjects with biliary AP was significantly higher in North America ( $45 \%$ vs. $14 \%$ for the remaining sites, $\mathrm{p}<0.001$ ). With respect to same admission cholecystectomy, considerable variations were noted among patients with mild acute biliary pancreatitis; it was performed in $60 \%$ of such patients in Latin America, while in only $15 \%$ in India $(\mathrm{p}<0.001)$. Moreover, early pancreatic interventions among patients with moderate or severe disease were more frequently performed in India (23\% vs. $7 \%$ in the remaining regions, $\mathrm{p}<0.001)$.

\section{Clinical Outcomes}


When comparing the LOS among mild AP, patients in North America were found to stay in the hospital the shortest time (4 days) compared to other regions (7 days; $\mathrm{p}<0.001)$. Severe AP developed in $23 \%$ of Indian patients compared to $9 \%$ in the rest of world ( $p<0.001$, Table 4$)$. ICU admissions were highest in Indian centers at 37.9\% (Table 5). In-hospital mortality was found to be the highest in Europe (5.7\%), followed by India (3.3\%), Latin America (2.3\%), and North America (0.6\%, $\mathrm{p}<0.001$, Table 5). Among European sites included, in hospital mortality in different countries was distributed as such; Greece: 0\%, Spain: $5 \%$, Lithuania: $6.4 \%$, and Romania: $8.6 \%$.

\section{Multivariate Analysis of outcomes:}

Based on multivariable regression analyses that adjusted for potential confounders such as age, gender, BMI, Charlson score, etiology, transfer status, and other factors, the odds of severe AP were 11.2 times higher in Europe [95\% confidence interval (CI): 5.8-21.6], 7 times higher in India (CI: 3.8-12.8), and 5.6 times higher in Latin America (CI: 2.8-11.1), compared to North America ( $\mathrm{p}<0.001$, Appendix Table 3). LOS was 4.3 days longer (CI: 3.5-5.4) in Europe, 1.1 days longer (CI: -0.1-2.3) in India, and 6.4 days longer (CI: 5.2-7.7) in Latin America when compared to North America ( $\mathrm{p}<0.001$, Appendix Table 4). The ORs for same-admission mortality among severe AP patients was 10.4 (CI: 2.7-40.5) in Europe, 4.2 (CI: 0.9-18.8) in India, and 8.3 (CI: 1.7-41.3) in Latin America when compared to North America $(\mathrm{p}<0.001$, Appendix Table 5).

\section{Discussion}

In this large prospectively collected registry, significant differences in AP patient demographics, etiology, management approaches, severity and clinical outcomes were seen around the world. 
Observed differences in etiology and demographics likely reflect a tight interconnection between age, gender, and etiology. In Indian sites, where the most preponderant AP etiology was alcohol, the majority of patients were young males. Previous studies have revealed a high proclivity of alcoholic pancreatitis in young Indian adults with heavy drinking patterns ${ }^{2,17-19}$. More specifically, a recent study from India published in 2018 reported an average age of 40 years with alcoholic pancreatitis representing $42 \%$ of all etiologies ${ }^{20}$. In Latin American sites, females were the predominant gender with biliary etiology being the most common. Latin America is known to have the highest rate of gallstone disease (more common among women) compared to other parts of the world ${ }^{21,22}$. A study in 2015 emanating from Argentina revealed similar findings, with biliary etiology accounting for $88 \%$ of all causes, and $58 \%$ of subjects being females $^{12}$. Along the same lines, older age among subjects from Europe is congruent with a study published in 2018 from this region ${ }^{13}$.

With regards to AP management, discrepancies in intravenous fluid volume and type administered over the first 24 hours are likely related to differences in accessibility to certain types of fluids, but most importantly, lack of high quality evidence supporting which type and what amount of fluid is optimal, as highlighted in the recent American Gastroenterological Association (AGA) guidelines in $2018^{23-27}$. Our findings further support the need for adequately powered, multi-center, randomized controlled trials comparing the efficacy of different fluid resuscitation protocols in AP patients.

The finding of disproportionally higher rate of opioid prescription during hospitalization and at the time of discharge in the North American sites is alarming. Of interest, a meta-analysis comparing NSAIDs versus opioids for pain control in AP subjects revealed no difference in the efficacy between the two treatments ${ }^{28,29}$. It not entirely clear why such divergences exist 
between North American centers compared to the rest of the world. Notably, no clear statements are included in the current societal guidelines addressing optimal strategies for analgesia in AP.

Based on strong evidence, current guidelines recommend limited utilization of urgent ERCP only among biliary AP patients with suspicion of cholangitis or biliary obstruction ${ }^{23}$. Our study showed that the rate of ERCPs performed in patients with biliary AP was much higher in North American sites. Impressive discrepancies have been previously reported in different counties, i.e. $81 \%$ in Hungary, $52 \%$ in the United States, and $9 \%$ in Argentina ${ }^{10-12}$. The discrepancies observed in our study are difficult to explain; they are possibly related to referral bias, local practice patterns, as well as compensation structure differences .

Recent evidence supports same admission cholecystectomy among patients with biliary AP ${ }^{23,24}$. Our study revealed that the rate of same admission cholecystectomy varied significantly with the highest seen in Latin America and lowest in India. Upon further discussion with site investigators, it appears that AP patients are traditionally admitted under surgical care in Latin America, making performance of inpatient cholecystectomy logistically easier. A recent publication from Latin America confirmed these findings, where 54\% of biliary AP subjects underwent same admission cholecystectomy ${ }^{12}$. In contrast, the low rate of same admission cholecystectomy in India could be explained by the high rate of transfers in the participating sites combined with patient preference to undergo this relatively simple operation locally at a later time.

Robust evidence highlights the use of enteral nutrition over TPN, and delaying pancreatic interventions in patients with moderate and severe AP, which is endorsed by current practice guidelines ${ }^{235,30,31}$. These recommendations were least adhered to in Indian centers, which is possibly accentuated by the higher rate of transfers. 
It is clear from the management practices seen in our study that the adherence to current evidence-driven societal guidelines varies significantly between different geographic regions of the world. Only a minority of the above practice patterns could be explained based on availability of resources. Thus, certain aspects of AP management such as the excessive administration of opioid analgesics and performance of ERCP in North American centers, overuse of TPN, and early pancreatic interventions in Indian sites, appear to lag behind the evidence. Additional effort is clearly needed to augment clinical implementation of certain therapeutic approaches supported by strong evidence in AP.

The finding that mild AP patients in North American centers had a shorter LOS compared to other regions is consistent with a recent report showing that the overall LOS of AP in the U.S. has decreased from 6.5 days in 1997 to 4.7 in $2015^{1}$. This is likely related to incentive policies that have been applied over the last two decades in the U.S. resulting in shortening inpatient $\operatorname{admissions}^{32}$.

Our study revealed higher death rate among European sites when compared to other geographic regions. . This observation could potentially be related to older age and higher rate of comorbid conditions seen in the European centers, both of which have been linked to mortality ${ }^{33}$. Notably, this difference persisted after adjustment for pertinent covariates in our multivariate analysis raising the question of other contributing factors. The lower mortality rate in North America seems consistent with recent reports indicating a decreased mortality over the last decade in the U.S, possibly related to improved quality of ICU care, and optimal timing for interventions ${ }^{14,34}$. Factors pertaining to baseline health and socioeconomic factors could possibly have contributed to these dispcrepancies in mortality. 
This study, has several strengths. It is the first of its kind to characterize differences in demographics, etiology, clinical profile, and management patterns and clinical outcomes in AP, by giving a snapshot of subject characteristics across different geographical regions of the world. Prior studies tackling this topic were limited by national bounds and lack of standardized methods for data acquisition. Distinctive attributes, which contribute to this study's strength, include its prospective nature, the large sample size with balanced representation between the different geographic areas with inclusion of at least 300 subjects from each studied region. Another important feature is the relatively recent time of data acquisition over the last 3 years, following the introduction of the RAC thus, accurately reflecting current practices ${ }^{7,24}$. Moreover, most included sites were large, reputable institutions, with a high degree of expertise relating to pancreatic diseases. Furthermore, data collection was standardized, under rigorous monitoring resulting in a high data completeness rate, and quality. Finally, at the conclusion of the data collection process, in an attempt to better understand regional practice patterns, an additional step was undertaken in obtaining site investigators' input into explaining the observed results.

With regards to the study's limitations, certain parts of the world such as Africa, the Middle East, or East Asia, were not represented. Moreover, the majority of participating sites were academic tertiary care hospitals, which may introduce a bias potentially affecting the generalizability of our results. Especially in North America, major ERCP referral centers were included whose unusual practice mix may not reflect that of the typical large American hospital. Finally, the proportion of subjects enrolled in the study compared to all AP patients hospitalized at each site, varied based on available research resources. 
In conclusion, we present a bird's eye view of the variations in clinical characteristics of AP patients across the world by using a large, prospective, international registry. There appears to be remarkable variations in frequency of AP etiologies in different regions. The therapeutic interventions specific to each region are in certain aspects strikingly divergent, and in many occasions lag behind current evidence. Outcomes, such as LOS and mortality, are largely variable. In addition to depicting key features of AP, the results from this study may serve as a reference guide for designing future clinical trials. 


\section{References:}

1. Peery AF, Crockett SD, Barritt AS, et al. Burden of Gastrointestinal, Liver, and Pancreatic Diseases in the United States. Gastroenterology 2015;149:1731-1741 e3.

2. Yadav D, Lowenfels AB. Trends in the epidemiology of the first attack of acute pancreatitis: a systematic review. Pancreas 2006;33:323-30.

3. Vidarsdottir $\mathrm{H}$, Moller $\mathrm{PH}$, Vidarsdottir $\mathrm{H}$, et al. Acute pancreatitis: a prospective study on incidence, etiology, and outcome. Eur J Gastroenterol Hepatol 2013;25:1068-75.

4. Easler JJ, de-Madaria E, Nawaz H, et al. Patients With Sentinel Acute Pancreatitis of Alcoholic Etiology Are at Risk for Organ Failure and Pancreatic Necrosis: A Dual-Center Experience. Pancreas 2016;45:997-1002.

5. Besselink MG, Verwer TJ, Schoenmaeckers EJ, et al. Timing of surgical intervention in necrotizing pancreatitis. Arch Surg 2007;142:1194-201.

6. van Santvoort HC, Besselink MG, Bakker OJ, et al. A step-up approach or open necrosectomy for necrotizing pancreatitis. N Engl J Med 2010;362:1491-502.

7. Banks PA, Bollen TL, Dervenis C, et al. Classification of acute pancreatitis--2012: revision of the Atlanta classification and definitions by international consensus. Gut 2013;62:102-11.

8. Agarwal S, George J, Padhan RK, et al. Reduction in mortality in severe acute pancreatitis: A time trend analysis over 16 years. Pancreatology 2016;16:194-9.

9. Munigala S, Yadav D. Case-fatality from acute pancreatitis is decreasing but its population mortality shows little change. Pancreatology 2016;16:542-50.

10. Parniczky A, Kui B, Szentesi A, et al. Prospective, Multicentre, Nationwide Clinical Data from 600 Cases of Acute Pancreatitis. PLoS One 2016;11:e0165309.

11. Koutroumpakis E, Slivka A, Furlan A, et al. Management and outcomes of acute pancreatitis patients over the last decade: A US tertiary-center experience. Pancreatology 2017;17:32-40.

12. Ocampo C, Kohan G, Leiro F, et al. Acta Gastroenterol Latinoam 2015;45:295-302.

13. Sternby H, Bolado F, Canaval-Zuleta HJ, et al. Determinants of Severity in Acute Pancreatitis: A Nation-wide Multicenter Prospective Cohort Study. Ann Surg 2018.

14. Yadav D, Lowenfels AB. The epidemiology of pancreatitis and pancreatic cancer. Gastroenterology 2013;144:1252-61.

15. Papachristou GI, Machicado JD, Stevens T, et al. Acute pancreatitis patient registry to examine novel therapies in clinical experience (APPRENTICE): an international, multicenter consortium for the study of acute pancreatitis. Ann Gastroenterol 2017;30:106-113.

16. Harris PA, Taylor R, Thielke $R$, et al. Research electronic data capture (REDCap)--a metadatadriven methodology and workflow process for providing translational research informatics support. J Biomed Inform 2009;42:377-81.

17. Yang AL, Vadhavkar S, Singh $\mathrm{G}$, et al. Epidemiology of alcohol-related liver and pancreatic disease in the United States. Arch Intern Med 2008;168:649-56.

18. Lankisch PG, Assmus C, Maisonneuve P, et al. Epidemiology of pancreatic diseases in Luneburg County. A study in a defined german population. Pancreatology 2002;2:469-77.

19. Erol A, Karpyak VM. Sex and gender-related differences in alcohol use and its consequences: Contemporary knowledge and future research considerations. Drug Alcohol Depend 2015;156:113.

20. Manrai M, Kochhar R, Gupta V, et al. Outcome of Acute Pancreatic and Peripancreatic Collections Occurring in Patients With Acute Pancreatitis. Ann Surg 2018;267:357-363.

21. Whitcomb DC, LaRusch J, Krasinskas AM, et al. Common genetic variants in the CLDN2 and PRSS1-PRSS2 loci alter risk for alcohol-related and sporadic pancreatitis. Nat Genet 2012;44:1349-54. 
22. Shaffer EA. Gallstone disease: Epidemiology of gallbladder stone disease. Best Pract Res Clin Gastroenterol 2006;20:981-96.

23. Crockett SD, Wani S, Gardner TB, et al. American Gastroenterological Association Institute Guideline on Initial Management of Acute Pancreatitis. Gastroenterology 2018;154:1096-1101.

24. Tenner S, Baillie J, DeWitt J, et al. American College of Gastroenterology guideline: management of acute pancreatitis. Am J Gastroenterol 2013;108:1400-15; 1416.

25. Wang MD, Ji Y, Xu J, et al. Early goal-directed fluid therapy with fresh frozen plasma reduces severe acute pancreatitis mortality in the intensive care unit. Chin Med J (Engl) 2013;126:19878.

26. Mao EQ, Fei J, Peng YB, et al. Rapid hemodilution is associated with increased sepsis and mortality among patients with severe acute pancreatitis. Chin Med J (Engl) 2010;123:1639-44.

27. Wu BU, Hwang JQ, Gardner TH, et al. Lactated Ringer's solution reduces systemic inflammation compared with saline in patients with acute pancreatitis. Clin Gastroenterol Hepatol 2011;9:710717 e1.

28. Peiro AM, Martinez J, Martinez E, et al. Efficacy and tolerance of metamizole versus morphine for acute pancreatitis pain. Pancreatology 2008;8:25-9.

29. Basurto Ona X, Rigau Comas D, Urrutia G. Opioids for acute pancreatitis pain. Cochrane Database Syst Rev 2013:CD009179.

30. Yi F, Ge L, Zhao J, et al. Meta-analysis: total parenteral nutrition versus total enteral nutrition in predicted severe acute pancreatitis. Intern Med 2012;51:523-30.

31. van Santvoort HC, Bakker OJ, Bollen TL, et al. A conservative and minimally invasive approach to necrotizing pancreatitis improves outcome. Gastroenterology 2011;141:1254-63.

32. Bueno $\mathrm{H}$, Ross JS, Wang $\mathrm{Y}$, et al. Trends in length of stay and short-term outcomes among Medicare patients hospitalized for heart failure, 1993-2006. JAMA 2010;303:2141-7.

33. de Beaux AC, Palmer KR, Carter DC. Factors influencing morbidity and mortality in acute pancreatitis; an analysis of 279 cases. Gut 1995;37:121-6.

34. McNabb-Baltar J, Ravi P, Isabwe GA, et al. A population-based assessment of the burden of acute pancreatitis in the United States. Pancreas 2014;43:687-91. 
Figure 1 legend: Centers' location and enrollment per center 
Table 1: Characteristics of Participating Centers

\begin{tabular}{|c|c|c|c|c|c|}
\hline Center & $\begin{array}{l}\text { Geographi } \\
\text { c Area }\end{array}$ & $\begin{array}{c}\text { Total } \\
\text { Enrolled }\end{array}$ & $\begin{array}{l}\text { Estimated } \\
\text { \# of Beds }\end{array}$ & $\begin{array}{l}\text { Estimated \# of AP } \\
\text { Admissions/Year }\end{array}$ & $\begin{array}{c}\text { Estimated } \\
\text { rate of } \\
\text { transfers }\end{array}$ \\
\hline LUHS, Kaunas, Lithuania & Europe & 109 & $>1000$ & $100-200$ & $50-75 \%$ \\
\hline University of Medicine, Cluj-Napoca, & Europe & 82 & $101-200$ & $50-100$ & $25-50 \%$ \\
\hline University of Medicine, Bucharest, Romania & Europe & 70 & $>1000$ & $100-200$ & $<25 \%$ \\
\hline Sapienza University, Rome, Italy & Europe & 69 & $301-500$ & $50-100$ & $<25 \%$ \\
\hline Attikon University, Athens, Greece & Europe & 59 & $501-750$ & $50-100$ & $<25 \%$ \\
\hline Investigación, Alicante, Spain & Europe & 20 & $751-1000$ & $100-200$ & $<25 \%$ \\
\hline AIG, Hyderabad, India & India & 136 & $201-300$ & $200-300$ & $>75 \%$ \\
\hline Postgraduate Institute, Chandigarh, India & India & 119 & $>1000$ & $300-500$ & $50-75 \%$ \\
\hline Apollo Gleneagles, Kolkata, India & India & 111 & $501-750$ & $50-100$ & $<25 \%$ \\
\hline UAN, Monterrey, Mexico & Latin & 95 & $301-500$ & $100-200$ & $<25 \%$ \\
\hline Hospital Nacional, Itaugua, Paraguay & Latin & 83 & $301-500$ & $100-200$ & $<25 \%$ \\
\hline Nacional "Posadas", Buenos Aires, Argentina & Latin & 71 & $301-500$ & $100-200$ & $<25 \%$ \\
\hline Universidad Autónoma, Mexico City, Mexico & Latin & 47 & $201-300$ & $50-100$ & $25-50 \%$ \\
\hline Hospital de Argudos, Buenos Aires, Argentina & Latin & 29 & $301-500$ & $50-100$ & $25-50 \%$ \\
\hline UPMC, Pittsburgh, USA & North & 130 & $751-1000$ & $100-200$ & $50-75 \%$ \\
\hline Johns Hopkins, Baltimore, USA & North & 90 & $>1000$ & $100-200$ & $25-50 \%$ \\
\hline Cleveland Clinic, Cleveland, USA & North & 82 & $>1000$ & $>500$ & $25-50 \%$ \\
\hline EMMC, Bangor, USA & North & 81 & $301-500$ & $100-200$ & $25-50 \%$ \\
\hline Indiana University, Indianapolis, USA & North & 62 & $201-300$ & $200-300$ & $50-75 \%$ \\
\hline AGH, Pittsburgh, USA & North & 32 & $501-750$ & $300-500$ & $25-50 \%$ \\
\hline MUSC, Charleston, USA & North & 18 & $751-1000$ & $200-300$ & $50-75 \%$ \\
\hline Kaiser, Los Angeles, USA & North & 17 & $301-500$ & $100-200$ & $<25 \%$ \\
\hline
\end{tabular}

AP: acute pancreatitis, LUHS: Lithuanian University of Health Sciences, AIG: Asian Institute of Gastroenterology,

UPMC: University of Pittsburgh Medical Center, UAN: Universidad Autónoma de Nueva , EMMC: Eastern Maine

Medical Center. 
Table 2: Comparison of AP patient demographics in different geographic regions.

\begin{tabular}{|c|c|c|c|c|c|c|}
\hline Variable & $\begin{array}{l}\text { Europe } \\
(\mathrm{n}=409)\end{array}$ & $\begin{array}{c}\text { India } \\
(n=366)\end{array}$ & $\begin{array}{c}\text { Latin } \\
\text { America } \\
(n=325)\end{array}$ & $\begin{array}{c}\text { North } \\
\text { America } \\
(n=512)\end{array}$ & $\begin{array}{c}\text { Total } \\
(n=1612)\end{array}$ & P Value \\
\hline Age, Median (IQR) & $58(45-74)$ & $39(30-50)$ & $43(29-59)$ & $52(37-65)$ & $49(34-64)$ & $<0.001$ \\
\hline Gender, Male (\%) & $203(49.6)$ & $274(74.9)$ & $108(33.5)$ & $258(50.6)$ & $843(52.5)$ & $<0.001$ \\
\hline $\begin{array}{l}\text { Ethnicity, Hispanic or } \\
\text { Latino }(\%)\end{array}$ & $3(0.7)$ & $0(0.0)$ & $303(97.4)$ & $20(4.0)$ & $326(20.6)$ & $<0.001$ \\
\hline \multicolumn{7}{|l|}{ Race (not Hispanic) } \\
\hline - $\quad$ Asian Indian (\%) & $2(0.5)$ & $361(99.2)$ & $0(0.0)$ & $6(1.2)$ & $36(29.3)$ & \multirow[t]{4}{*}{$<0.001$} \\
\hline $\begin{array}{cl}- & \text { Black or African - } \\
& \text { American }(\%)\end{array}$ & $0(0.0)$ & $0(0.0)$ & $0(0.0)$ & $82(16.9)$ & $82(6.5)$ & \\
\hline - $\quad$ White $(\%)$ & $397(99.3)$ & $0(0.0)$ & $8(100.0)$ & $386(79.4)$ & $791(62.9)$ & \\
\hline - $\quad$ Others $(\%)$ & $1(0.3)$ & $3(0.8)$ & $0(0.0)$ & $12(2.5)$ & $16(1.3)$ & \\
\hline CCI >1(\%) & $298(72.9)$ & $132(36.1)$ & $153(47.1)$ & $314(61.3)$ & 897 (55.6) & $<0.001$ \\
\hline Obesity, BMI $\geq 30(\%)$ & $111(28.5)$ & $27(7.4)$ & $86(27.0)$ & $220(43.3)$ & $444(28.0)$ & $<0.001$ \\
\hline \multicolumn{7}{|l|}{ Etiology } \\
\hline - $\quad$ Biliary (\%) & $206(50.4)$ & $102(27.9)$ & $249(78.1)$ & $170(33.3)$ & $727(45.3)$ & \multirow[t]{6}{*}{$<0.001$} \\
\hline - $\quad$ Alcohol (\%) & $78(19.1)$ & $163(44.5)$ & $6(1.9)$ & $89(17.5)$ & $336(20.9)$ & \\
\hline - $\quad$ Idiopathic (\%) & $74(18.1)$ & $77(21.0)$ & $22(6.9)$ & $92(18.0)$ & $265(16.5)$ & \\
\hline $\begin{array}{l}\text { Hypertriglyceride } \\
\text { mia }(\%)\end{array}$ & $19(4.6)$ & $7(1.9)$ & $19(6.0)$ & $30(5.9)$ & $75(4.7)$ & \\
\hline - $\quad$ Post-ERCP $(\%)$ & $13(3.2)$ & $8(2.2)$ & $15(4.7)$ & $97(19.0)$ & $133(8.3)$ & \\
\hline - $\quad$ Other $(\%)$ & $19(4.6)$ & $9(2.5)$ & $8(2.5)$ & $32(6.3)$ & $68(4.2)$ & \\
\hline Current smoking & $103(26.1)$ & $95(26.0)$ & $38(11.9)$ & $129(25.3)$ & $365(23.0)$ & $<0.001$ \\
\hline Current alcohol use & $194(49.1)$ & $166(45.4)$ & $57(17.9)$ & $189(37.1)$ & $606(38.1)$ & $<0.001$ \\
\hline Recurrent AP & $95(23.2)$ & $75(20.5)$ & $42(13.2)$ & $185(36.3)$ & $397(24.8)$ & $<0.001$ \\
\hline Transfers (\%) & $81(19.8)$ & $260(71.0)$ & $35(11.0)$ & $171(33.5)$ & $547(34.1)$ & $<0.001$ \\
\hline
\end{tabular}

AP: acute pancreatitis, IQR: inter-quartile range, CCI: charlson comorbidity index, BMI: Body mass index. $P$ values were calculated based on Fisher's exact for categorical variables and Kruskal-Wallis global tests for continuous variables. Overall data completion rate was more than 95\% for each of the variables 
Table 3: Comparison of AP management practices in different regions.

\begin{tabular}{|c|c|c|c|c|c|c|}
\hline Variable & $\begin{array}{l}\text { Europe } \\
(\mathrm{n}=409)\end{array}$ & $\begin{array}{c}\text { India } \\
(n=366)\end{array}$ & $\begin{array}{c}\text { Latin } \\
\text { America } \\
(n=325)\end{array}$ & $\begin{array}{c}\text { North } \\
\text { America } \\
(\mathrm{n}=512)\end{array}$ & $\begin{array}{c}\text { Total } \\
(n=1612)\end{array}$ & $\mathrm{P}$ value \\
\hline \multicolumn{7}{|l|}{ Intravenous fluids } \\
\hline $\begin{array}{cc}\text { - } & \text { Amount, median } \\
(\mathrm{IQR})^{*} & \end{array}$ & $2.5(2.0-3.6)$ & $3.2(2.0-4.5)$ & $\begin{array}{c}3.0(2.5- \\
3.8)\end{array}$ & $\begin{array}{c}3.0(2.0- \\
4.2)\end{array}$ & $3.0(2.0-4.0)$ & $<0.001$ \\
\hline $\begin{array}{l}-\quad \text { Type of fluid, LR } \\
(\%)\end{array}$ & $315(77.0)$ & $337(92.3)$ & $24(7.4)$ & $253(49.4)$ & $930(57.7)$ & $<0.001$ \\
\hline \multicolumn{7}{|l|}{ Inpatient pain management } \\
\hline$-\quad$ NSAIDs $(\%)$ & 277 (67.7) & $1(0.3)$ & $155(47.7)$ & $91(17.8)$ & $524(32.5)$ & $<0.001$ \\
\hline - $\quad$ Tramadol $(\%)$ & $184(45.0)$ & $334(91.3)$ & $111(34.2)$ & $40(7.8)$ & $669(41.5)$ & $<0.001$ \\
\hline - $\quad$ Opioids $(\%)$ & $41(11.9)$ & $90(24.9)$ & $167(59.0)$ & $454(92.5)$ & $752(50.8)$ & $<0.001$ \\
\hline Opioids at discharge (\%) & $1(0.3)$ & $2(0.6)$ & $17(6.2)$ & $314(64.3)$ & $334(23.3)$ & $<0.001$ \\
\hline \multicolumn{7}{|l|}{ Nutritional support } \\
\hline $\begin{array}{ll}-\quad & \text { Enteral Nutrition } \\
& (\%)^{* *}\end{array}$ & $34(31.8)$ & $43(19.9)$ & $15(15.3)$ & $46(34.8)$ & $138(25.0)$ & $<0.001$ \\
\hline$-\quad \mathrm{TPN}(\%)^{* *}$ & $3(2.8)$ & $59(27.3)$ & $4(4.1)$ & $9(6.8)$ & $75(13.6)$ & $<0.001$ \\
\hline $\operatorname{ERCP}(\%)^{¥}$ & $29(14.4)$ & $17(16.8)$ & $34(14.1)$ & $76(44.7)$ & $156(21.9)$ & $<0.001$ \\
\hline Cholecystectomy $(\%)^{\ddagger}$ & $52(31.7)$ & $6(15.0)$ & $101(59.8)$ & $52(42.6)$ & $211(42.6)$ & $<0.001$ \\
\hline $\begin{array}{l}\text { Early pancreatic intervention } \\
(\%)^{* *}\end{array}$ & $9(8.4)$ & $50(23.1)$ & $5(5.1)$ & $9(6.8)$ & $73(13.2)$ & $<0.001$ \\
\hline
\end{tabular}

LR: Lactated ringers; NSAIDs: Non steroidal Anti-Inflammatory Drugs. P values are based on Fisher's exact for categorical variables and Kruskall-Wallis global tests for continuous one. *Amount in liters within initial 24 hours of admission. ** Among RAC moderately severe or severe patients. $¥$ Among Biliary AP patients. $¥$ Among RAC mild biliary AP patients.

Missing data: Narcotics use during hospitalization was missing in 65 patients in Europe, 4 in India, 23 in Latin America and 21 subjects in North America. Overall data completion rate for narcotics during hospitalization was $91.8 \%$.

Narcotics at discharge were missing in 90 patients in Europe, 16 in India, 51 in Latin America and 24 subjects in North America. The overall data completion rate for Narcotics at discharge was $88.8 \%$; all other variables had overall data completion rate of over $95 \%$. 
Table 4: Comparison of AP severity in various regions of the world.

\begin{tabular}{|l|c|c|c|c|c|c|}
\hline Severity based on RAC & $\begin{array}{c}\text { Europe } \\
(\mathrm{n}=409)\end{array}$ & $\begin{array}{c}\text { India } \\
(\mathrm{n}=366)\end{array}$ & $\begin{array}{c}\text { Latin America } \\
(\mathrm{n}=325)\end{array}$ & $\begin{array}{c}\text { North } \\
\text { America } \\
(\mathrm{n}=512)\end{array}$ & $\begin{array}{c}\text { Total } \\
(\mathrm{n}=1612) \\
\text { Value* }\end{array}$ \\
\hline -Mild (\%) & $296(73.4)$ & $148(40.7)$ & $213(68.5)$ & $374(73.9)$ & $1031(65.1)$ & $<0.001$ \\
\hline -Mod. severe (\%) & $59(14.6)$ & $134(36.8)$ & $75(24.1)$ & $94(18.6)$ & $362(22.9)$ \\
\hline -Severe (\%) & $48(11.9)$ & $82(22.5)$ & $23(7.4)$ & $38(7.5)$ & $191(12.1)$ \\
\hline
\end{tabular}

RAC: revised Atlanta classification. Data completion rate is more than $95 \%$.

* Fisher's exact test was used as a global test to assess the association between regions and RAC severity. 
Table 5: Comparison of AP LOS, ICU admissions, and in hospital mortality among various regions within each RAC group and among all study participants.

\begin{tabular}{|c|c|c|c|c|c|c|}
\hline \multicolumn{7}{|l|}{ LOS } \\
\hline LOS per RAC groups & $\begin{array}{l}\text { Europe } \\
(n=409)\end{array}$ & $\begin{array}{c}\text { India } \\
(n=366)\end{array}$ & $\begin{array}{l}\text { Latin America } \\
\qquad(n=325)\end{array}$ & $\begin{array}{c}\text { North } \\
\text { America } \\
(n=512)\end{array}$ & $\begin{array}{c}\text { Total } \\
(n=1612)\end{array}$ & P Value \\
\hline -Mild AP, median (IQR) & $7(6-10)$ & $7(5-9)$ & $10(6-16)$ & $4(3-6)$ & $6(4-10)$ & $<0.001^{*}$ \\
\hline -Mod. severe, median (IQR) & $11(8.5-18)$ & $10(7-15)$ & $17(8 .-26)$ & $8.0(6-12.8)$ & $11(7-16)$ & $<0.001 *$ \\
\hline -Severe, median (IQR) & $28(25-41)$ & $19(13-25)$ & $19(13-25)$ & $20(13.5-32.5)$ & $20(14-31)$ & $<0.001 *$ \\
\hline -Overall, median (IQR) & $8(6-12)$ & $9(6-15)$ & $11(7-19)$ & $5(3-8)$ & $8(5-13)$ & $<0.001 * *$ \\
\hline \multicolumn{7}{|l|}{ ICU Admissions } \\
\hline ICU per RAC groups & $\begin{array}{l}\text { Europe } \\
(\mathrm{n}=409)\end{array}$ & $\begin{array}{c}\text { India } \\
(n=366)\end{array}$ & $\begin{array}{l}\text { Latin America } \\
\qquad(\mathrm{n}=325)\end{array}$ & $\begin{array}{l}\text { North America } \\
\qquad(\mathrm{n}=512)\end{array}$ & $\begin{array}{c}\text { Total } \\
(n=1612)\end{array}$ & P Value \\
\hline -Mild AP (\%) & $2(0.7)$ & $18(12.2)$ & $0(0.0)$ & $9(2.4)$ & $29(2.8)$ & $<0.001 ¥$ \\
\hline -Mod. severe (\%) & $11(18.6)$ & $54(40.3)$ & $3(4.0)$ & $26(27.7)$ & $93(25.8)$ & $<0.001 ¥$ \\
\hline -Severe AP (\%) & $39(81.2)$ & $66(80.5)$ & $10(43.5)$ & $33(86.8)$ & $148(77.5)$ & $<0.001 ¥$ \\
\hline -Overall (\%) & $54(13.3)$ & $138(37.9)$ & $13(4.2)$ & $68(13.4)$ & $273(17.2)$ & $<0.001 ¥ ¥$ \\
\hline \multicolumn{7}{|l|}{ In Hospital Morality } \\
\hline $\begin{array}{l}\text { Mortality in various RAC } \\
\text { groups }\end{array}$ & $\begin{array}{l}\text { Europe } \\
(\mathrm{n}=409)\end{array}$ & $\begin{array}{c}\text { India } \\
(n=366)\end{array}$ & $\begin{array}{l}\text { Latin America } \\
\qquad(\mathrm{n}=325)\end{array}$ & $\begin{array}{l}\text { North America } \\
\qquad(\mathrm{n}=512)\end{array}$ & $\begin{array}{c}\text { Total } \\
(n=1612)\end{array}$ & P Value \\
\hline -Mild AP (\%) & $0(0.0)$ & $0(0.0)$ & $0(0.0)$ & $0(0.0)$ & $0(0.0)$ & NA \\
\hline -Mod. severe (\%) & $2(3.4)$ & $1(0.8)$ & $0(0.0)$ & $0(0.0)$ & $3(0.8)$ & $0.12 \mathbb{I}$ \\
\hline -Severe AP (\%) & $21(43.8)$ & $11(13.4)$ & $7(30.4)$ & $3(7.9)$ & $42(28.2)$ & $<0.001 \Phi$ \\
\hline -Overall (\%) & $23(5.7)$ & $12(3.3)$ & $7(2.3)$ & $3(0.6)$ & $45(2.8)$ & $<0.001 \mathrm{dTI}$ \\
\hline
\end{tabular}

LOS: length of stay. Mod. severe: moderately severe; ICU: intensive care unit; RAC: revised Atlanta criteria. Data completion rate is more than $95 \%$.

* Kruskal-Wallis test was used to assess the association between regions and LOS within different severity groups.

* * Kruskal-Wallis test was also applied for the association between regions and LOS among all participants.

$¥$ Fisher’s exact test was used to assess the association between regions and ICU admissions within different severity groups.

$¥ ¥$ Fisher’s exact test was also applied for the association between regions and ICU admissions among all study participants. 
II Fisher's exact test was used to assess the association between region and mortality (assessed in moderately severe and severe groups; no death seen in mild AP group)

III Fisher's exact test was also applied for the association between hospital mortality and regions among all study participants. 


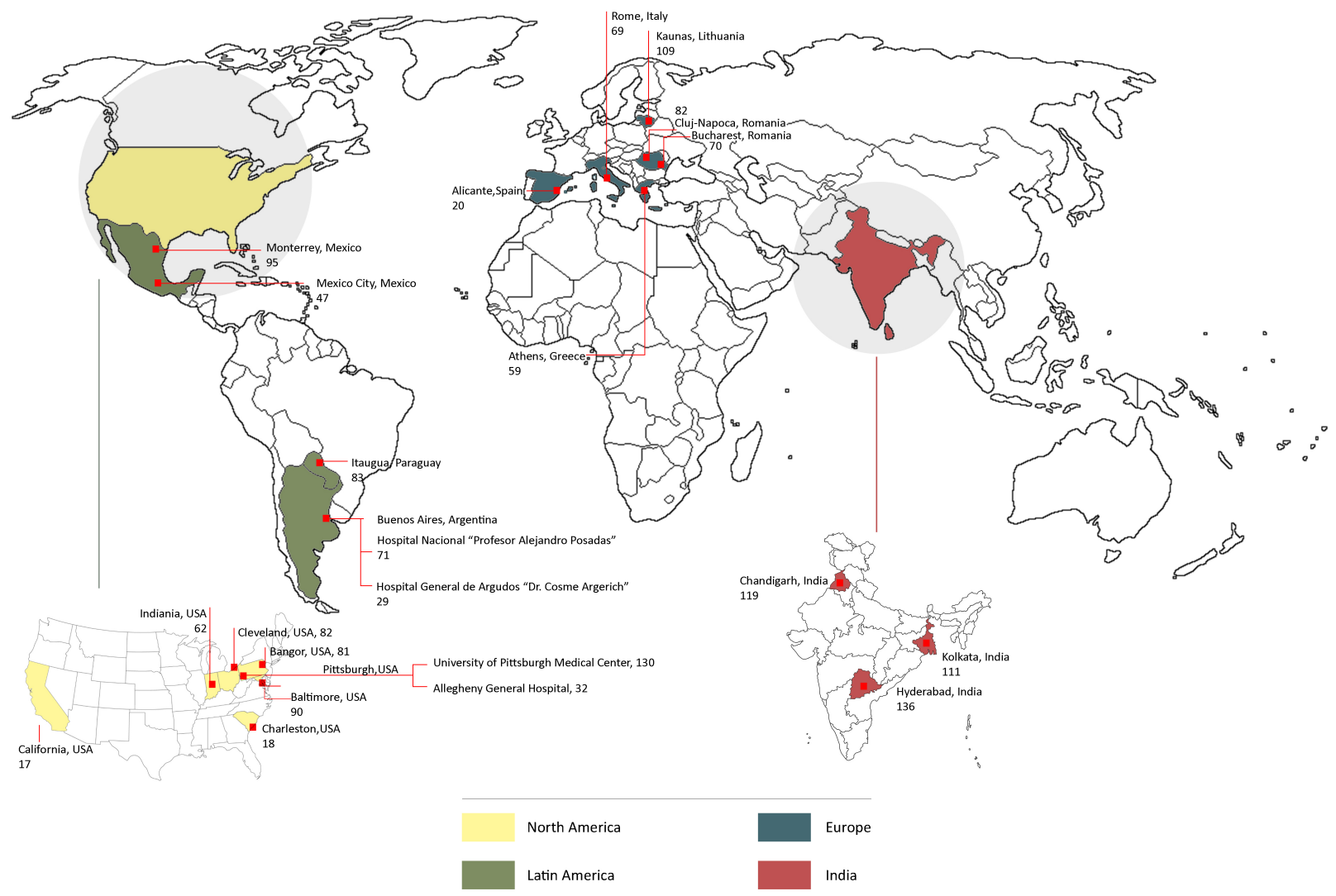


Appendix Table 1. Study questionnaire.

(see attached PDF folder) 
Appendix table 2: Definitions of collected variables.

\begin{tabular}{|c|c|}
\hline Variable & Definition \\
\hline AP diagnosis & $\begin{array}{l}\text { At least } 2 \text { out } 3 \text { three criteria: } \\
\text { 1) upper abdominal pain characteristic of AP } \\
\text { 2) serum amylase and/or lipase } \geq 3 \text { times the upper limit of normal } \\
\text { 3) imaging findings characteristic of AP }\end{array}$ \\
\hline Current smoking & Active smoking within 6 months prior to admission \\
\hline Current alcohol use & AP preceded by heavy alcohol consumption as determined by site investigators \\
\hline Alcoholic AP & AP preceded by heavy alcohol consumption as determined by site investigators \\
\hline Biliary AP & $\begin{array}{l}\text { AP with objective evidence of cholelithiasis or choledocholithiasis on imaging, and } \\
\text { no other plausible explanation for pancreatitis as determined by site investigators }\end{array}$ \\
\hline $\begin{array}{l}\text { Hypertriglyceridemia } \\
\text { induced AP }\end{array}$ & $\begin{array}{l}\text { AP occurring in setting of a high serum triglyceride level }(>500 \mathrm{mg} / \mathrm{dL}) \text { with } \\
\text { exclusion of other causes. Post ERCP AP: development of AP within } 24 \text { hours of } \\
\text { ERCP }\end{array}$ \\
\hline Other cause of AP & AP with the presence of a clear inciting factor, such as a suspected medication. \\
\hline Idiopathic AP & AP not fitting any of the above mentioned categories \\
\hline $\begin{array}{l}\text { Early pancreatic } \\
\text { interventions }\end{array}$ & $\begin{array}{l}\text { Open surgical, minimally invasive, endoscopic, or percutaneous approaches in } \\
\text { drainage or debridement, performed within } 2 \text { weeks of admission }\end{array}$ \\
\hline Organ Failure & $\begin{array}{l}\text { Score }>1 \text { on the modified Marshal system for cardiovascular, pulmonary, or renal } \\
\text { failure }\end{array}$ \\
\hline Time of admission & $\begin{array}{l}\text { Time of index presentation to hospital; in cases where subjects were transferred } \\
\text { from outside hospitals, time of admission referred to the original presentation to the } \\
\text { hospital, and total LOS included the duration of stay in both the primary and referra } \\
\text { center }\end{array}$ \\
\hline Enteral nutrition & Nutrition by means of a feeding tube (nasogastric or nasojejunal) \\
\hline Parenteral nutrition & $\begin{array}{l}\text { Intravenous nutrition (subjects who received both enteral and parenteral nutrition } \\
\text { were categorized as having received parenteral nutrition) }\end{array}$ \\
\hline Mortality & Death during the same hospitalization \\
\hline $\begin{array}{l}\text { Systemic } \\
\text { inflammatory } \\
\text { response syndrome }\end{array}$ & $\begin{array}{l}\text { Positive when at least } 2 \text { of the following criteria were present: } \\
\text { 1) Heart rate }>90\end{array}$ \\
\hline
\end{tabular}




\begin{tabular}{|l|l|}
\hline & $\begin{array}{l}\text { 2) Body temperature }>38 \square \text { or }<36 \\
\text { 3) White blood cell count }>12000 / \mathrm{mm} 3 \text { or }<4000 / \mathrm{mm} 3 \\
\text { 4) Respiratory rate }>20\end{array}$ \\
\hline
\end{tabular}

AP: acute pancreatitis; LOS: length of stay. 
Appendix table 3. Multivariate logistic regression model that compares severity of AP (severe AP vs. mild/moderately severe APs) among regions

\begin{tabular}{|c|c|c|}
\hline Variables & OR (95\% CI) & p-value \\
\hline $\begin{array}{c}\text { Regions (vs. North } \\
\text { America) }\end{array}$ & & $<0.001^{*}$ \\
\hline Europe & $11.2(5.8,21.6)$ & $<0.01$ \\
\hline India & $7.0(3.8,12.8)$ & $<0.01$ \\
\hline Latin America & $5.6(2.8,11.1)$ & $<0.01$ \\
\hline Age & $1.0(1.0,1.0)$ & 0.28 \\
\hline Gender (Male) & $1.9(1.2,2.8)$ & $<0.01$ \\
\hline BMI (>=30) & $1.4(0.9,2.1)$ & 0.13 \\
\hline Charlson score (>1) & $0.7(0.4,1.3)$ & 0.29 \\
\hline $\begin{array}{c}\text { Etiology (vs. Biliary) } \\
\text { Alcoholic }\end{array}$ & $1.5(0.9,2.5)$ & $0.045^{*}$ \\
\hline Post-ERCP & $1.2(0.5,2.8)$ & 0.11 \\
\hline Other & $1.0(0.6,1.7)$ & 0.67 \\
\hline Transfer (Yes) & $5.8(3.7,9.0)$ & 0.91 \\
\hline $\begin{array}{c}\text { Cholecystectomy } \\
\text { (Yes) }\end{array}$ & $0.3(0.1,0.7)$ & $<0.01$ \\
\hline Opioid Use (Yes) & $5.2(3.4,8.1)$ & $<0.01$ \\
\hline
\end{tabular}

* The likelihood ratio tests were used for the association between severity of AP and factors with more than 2 categories (region and etiology).

A backward model selection procedure was followed 
Appendix table 4. Multivariable linear regression model that compares length of stay (LOS) among regions.

\begin{tabular}{|c|c|c|}
\hline Variable & Beta $(95 \%$ CI $)$ & p-value \\
\hline Regions (vs. NA) & & $<0.001 *$ \\
\hline $\mathrm{EU}$ & $4.3(3.3,-5.4)$ & $<0.01$ \\
\hline IND & $1.1(-0.1,-2.3)$ & 0.07 \\
\hline LA & $6.4(5.2,7.7)$ & $<0.01$ \\
\hline Age & $0.0(0.0,0.0)$ & 0.79 \\
\hline Gender (Male) & $0.2(-0.6,1.1)$ & 0.94 \\
\hline $\mathrm{BMI}(>=30)$ & $0.1(-0.8,1.0)$ & 0.45 \\
\hline Charlson score $(>1)$ & $0.1(-1.2,1.4)$ & 0.57 \\
\hline Etiology (vs Biliary) & & $0.02 *$ \\
\hline Alcoholic & $0.9(-0.3,2.2)$ & 0.13 \\
\hline Post-ERCP & $0.1(-1.4,1.6)$ & 0.87 \\
\hline Other & $0.3(-0.8,1.3)$ & 0.59 \\
\hline Transfer (Yes) & $2.2(1.3,3.1)$ & $<0.01$ \\
\hline $\begin{array}{c}\text { Cholecystectomy } \\
\text { (Yes) }\end{array}$ & $4.6(3.5,5.8)$ & $<0.01$ \\
\hline RAC (vs Moderate) & & $<0.01 *$ \\
\hline Mild & $-5.6(-6.6,-4.7)$ & $<0.01$ \\
\hline Severe & $10.6(9.1,12.0)$ & $<0.01$ \\
\hline
\end{tabular}

* The likelihood ratio tests were used for the association between LOS of AP and factors with more than 2 categories (region etiology and severity).

A backward model selection procedure was followed 
Appendix table 5. Multivariable logistic regression that compares mortality in patients with severe AP among regions.

\begin{tabular}{|c|c|c|}
\hline Variables & OR $(\mathbf{9 5 \%}$ CI) & p-value \\
\hline Regions (vs NA) & & $<0.001^{*}$ \\
\hline EU & $10.4(2.7,40.5)$ & 0.06 \\
\hline IND & $4.2(0.9,18.8)$ & $<0.01$ \\
\hline LA & $8.3(1.7,41.3)$ & $<0.01$ \\
\hline Age & $1.0(1.0,1.1)$ & 0.02 \\
\hline Gender (Male) & $1.4(0.6,3.4)$ & 0.46 \\
\hline BMI (>=30) & $2.0(0.8,5.0)$ & 0.13 \\
\hline Charlson score $(>1)$ & $1.2(0.3,4.5)$ & 0.65 \\
\hline
\end{tabular}

* The likelihood ratio test was used for the association between mortality of AP and regions.

A backward model selection procedure was followed 


\section{Demographics}

Record ID

All variables in brackets should preferably be obtained by interviewing the patient

Patient initials

$\overline{\text { (First name initial, last name initial) }}$

Age

Gender

(Age in years)

\{Race\}

$\bigcirc$ Female

$\bigcirc$ Male

White or Caucasian (not Hispanic)

Hispanic or Latino

$\bigcirc$ Native American

$\bigcirc$ Black or African American

$\bigcirc$ Asian Indian

Asian Oriental

$\bigcirc$ Asian Middle East

$\bigcirc$ Native Hawaiian or Other Pacific Islander

$\bigcirc$ Other

\{Weight $(\mathrm{kg})$

(Patient weight in kilograms)

\{Height $\}(\mathrm{cm})$

(Patient height in centimeters)

BMI (Body Mass Index)

\{Waist size (inches)

(The waist size can be estimated based on the patient's pants size. Use the following chart to transform the pants size to waist size in $\mathrm{cm}$.) 
waist size chart
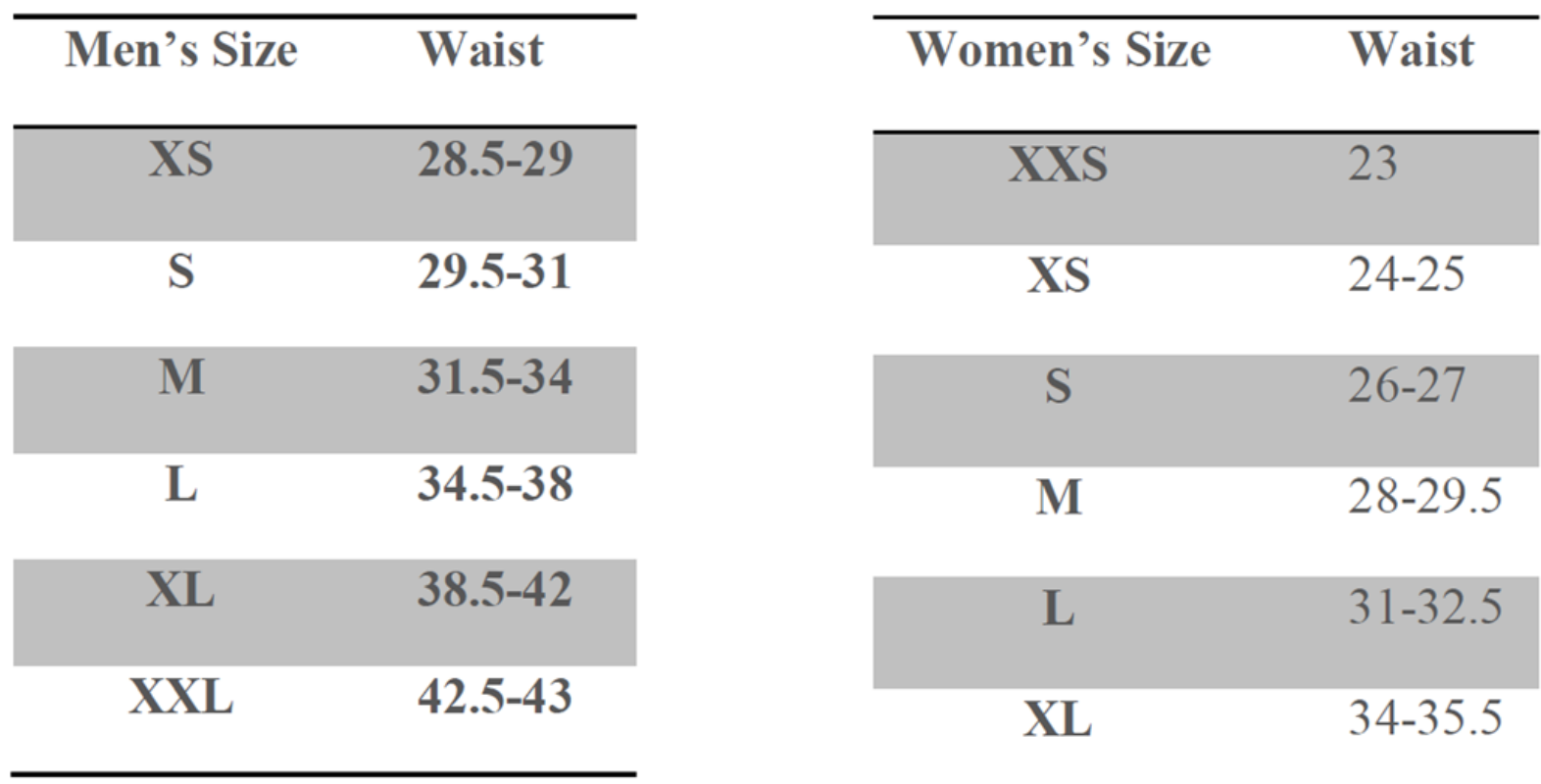

$\mathbf{L}$

$31-32.5$

XL

$34-35.5$

XXL

\section{History of Present Illness (Acute Pancreatitis)}

\{Date and time of Pain Onset\}

$\{$ Date and time of initial presentation to the hospital\}

Transfer

Date and time of admission to referral center
(Date and time when the characteristic upper abdominal pain of acute pancreatitis started)

(Date and time of initial presentation to emergency room, or direct admission to hospital)

$\bigcirc$ Yes

No

(It applies when the patient transfers from the hospital where he/she initially presented to a different hospital (referral center) for further management) 
Acute Pancreatitis primary etiology

Other cause

Is there any secondary etiology?

Acute Pancreatitis secondary etiology
Gallstones

Alcoholic

Idiopathic

$\bigcirc$ Hypertriglyceridemia-induced

$\bigcirc$ Post-ERCP (Endoscopic Retrograde Cholangiopancreatography)

Other

(Select the most prominent etiology. Idiopathic acute pancreatitis is defined as of no clear etiology after laboratory work-up has been completed and other common etiologies have been excluded; Hypertriglyceridemia-induced acute pancreatitis is confirmed when common etiologies have been excluded and serum triglycerides are $>500 \mathrm{mg} / \mathrm{dL}$ )

(Please write the other etiology responsible for causing Acute Pancreatitis)

$\bigcirc$ Yes

$\bigcirc$ No

Gallstones

Alcoholic

Idiopathic

Hypertriglyceridemia-induced

$\bigcirc$ Post-ERCP (Endoscopic Retrograde Cholangiopancreatography)

$\bigcirc$ Other

(ERCP stands for Endoscopic Retrograde Cholangiopancreatography)

Date and time of ERCP in the case of post-ERCP Acute Pancreatitis

Yes

No

(This refers to even single dose of Non Steroidal Anti Inflammatory Drugs (NSAIDS) taken within the last 7 days from the onset of acute pancreatitis. NSAIDS include aspirin, ibuprofen, indomethacin, naproxen, celecoxib, ketorolac, diclofenac, sulindac, etc)

Statin use

Yes

No

(This refers to daily use of statin before the onset of acute pancreatitis. Statins include atrovastatin, simvastatin, pravastatin, fluvastatin, etc)

Medications within the last one month
(Please write down the names of medications which were started within the last one month prior to pain onset.) 


\section{Past Medical History}

History of Acute Pancreatitis

\{Number of prior acute pancreatitis episodes\}

Prior cholecystectomy

History of Pre-existing Hypertriglyceridemia

Baseline Triglyceride (TG) level

Preexisting Diabetes Mellitus (DM)

Diabetes Mellitus (DM) type

End-organ damage due to Diabetes Mellitus

Congestive Heart Failure (CHF)

Myocardial infarction

Peripheral artery disease

Cerebrovascular disease
First episode

Recurrent episode (at least one episode before)

$\bigcirc$ Yes

No

$\bigcirc$ Yes

No

(Presence of Hypertriglyceridemia before onset of acute pancreatitis.)

(TG levels before this episode of acute pancreatitis (if TG level measurements are available from prior admissions or visits))

$\bigcirc$ Yes

No

(Presence of diabetes mellitus before the onset of acute pancreatitis)

Type 1

$\bigcirc$ Type 2 non-insulin dependent/diet controlled

Type 2 non-insulin dependent/on antidiabetics

$\bigcirc$ Type 2 insulin dependent

(Type of preexisting diabetes mellitus)

Yes

$\bigcirc$ No

(End organ damage includes retinopathy, neuropathy, or nephropathy)

$\bigcirc$ Yes

No

(Symptomatic congestive heart failure, i.e. NYHA functional class $\geq$ III)

Yes

No

(History of medically documented myocardial infarction)

Yes

No

(History of intermittent claudication, peripheral arterial bypass for insufficiency, gangrene, acute arterial insufficiency, untreated aortic aneurysm $(>=6 \mathrm{~cm})$ )

Yes

$\bigcirc$ No

(History of Transient Ischemic Attack (TIA), or Cerebral Vascular Attack (stroke) with no or minor sequelae) 
Dementia

Chronic pulmonary disease

Connective tissue disorders

Peptic Ulcer Disease (PUD)

Liver disease

Renal disease

Hemiplegia (or paraplegia)

Solid tumor

Metastasis of solid tumor

Leukemia
Yes

No

(Chronic cognitive deficit, i.e. Mini-Mental

Status Exam(MMSE) $\leq 26$ )

Yes

No

(Symptomatic dyspnea due to chronic respiratory conditions (including asthma))

$\bigcirc$ Yes

No

(Connective tissue disorders include Lupus,

Polymyositis, mixed Connective Tissue Disorders, Polymyalgia Rheumatica, moderate to severe Rheumatoid Arthritis)

Yes

No

(Patients who have required treatment for peptic ulcer disease)

$\bigcirc$ No

Mild

Moderate to severe

(Mild means chronic liver disease with/without compensated cirrhosis. Moderate to severe means decompensated cirrhosis (includes: ascites, portosystemic encephalopathy, or history of variceal bleeding))

No

Mild

Moderate to severe

(Mild means $\mathrm{Cr}>1.5 \mathrm{mg} / \mathrm{dL}$ ( $133 \mu \mathrm{mol} / \mathrm{L})$ and less than $3 \mathrm{mg} / \mathrm{dL}(265 \mu \mathrm{mol} / \mathrm{L})$. Moderate to severe means creatinine $>3 \mathrm{mg} / \mathrm{dL}(265 \mu \mathrm{mol} / \mathrm{L})$, history of renal transplantation, history of dialysis or history of uremic syndrome )

$\bigcirc$ Yes

No

(Hemiplegia means impairment in motor function of one side of the body. Papaplegia means impairment in motor function of lower extrimities.)

$\bigcirc$ Yes

$\bigcirc$ No

(Tumors diagnosed within the last 5 years (pancreatic cancers, non-melanomatous skin cancers, and in situ cervical carcinomas are excluded))

$\bigcirc$ Yes

No

$\bigcirc$ Yes

No

(Including chronic myeloid leukemia, chronic lymphocytic leukemia, acute myeloid leukemia, acute lymphocytic leukemia, polycytemia vera) 
Lymphoma

AIDS (not just positive HIV test) $\bigcirc$ Yes

No

(Including Non-Hodgkin, Hodgkin, Waldenstrom macroglobulinemia, Multiple Myeloma )

Yes

No

(Acquired Immune Deficiency Syndrome (AIDS) defined as confirmed positive Human Immunodeficiency Virus (HIV) test plus either CD4 count $<250$ or any HIV-related complications )

\section{Social History}

\{Smoking $\}$

$\{$ Total years of smoking $\}$

\{Average number of cigarettes per day\}

\{Alcohol consumption\}

\{Date/time of last drink\}

\{Total years of alcohol consumption $\}$

\{Average drinking days per week\}

\{Average drinks on a drinking day\}
Never (< 100 cigarettes or 5 packs in lifetime)

Active (within the last 6 months)

$\bigcirc$ Former ( $>6$ months without smoking)

$\overline{\text { (How many years has the patient }}$ smoked in total?)

$\overline{\text { (How many cigarettes on average }}$ does/did the patient smoke per day? )

Never ( $<20$ drinks in lifetime)

Active (within the last 6 months)

Former (>6 months without drinking)

(Does the patient drink alcohol?)

(When was the last alcoholic drink?)

(How many days on average does/did the patient drink per week?)

(How many drinks on average does/did the patient drink on a drinking day?)

\section{Family History}

Family history of Acute Pancreatitis

Family history of Chronic Pancreatitis $\bigcirc$ Yes

No

(Does the patient have any first-degree relatives diagnosed with acute pancreatitis (first-degree relatives include parents, siblings, or children) )

$\bigcirc$ Yes

No

(Does the patient have any first-degree relatives diagnosed with chronic pancreatitis (first-degree relatives include parents, siblings, or children) ) 
Family history of Cystic Fibrosis (CF)

Yes

No

(Does the patient have any first-degree relatives diagnosed with cystic fibrosis (first-degree

relatives include parents, siblings, or children) ) 


\section{On Admission}

Record ID

\section{Vital Signs}

Temperature on admission ( in Celsius, with 1 decimal)

(Temperature measured upon presenting to initial hospital (not transferred hospital))

Heart rate on admission (beats/min)

(Heart rate recorded upon presenting to initial hospital (not transferred hospital))

Respiratory rate on admission (breaths/min)

(Respiratory rate recorded upon presenting to initial hospital (not transferred hospital))

\section{Physical Examination}

Pain on admission

Nausea/vomiting on admission

Rebound tenderness/guarding on admission

Altered mental status on admission

Pleural effusions assessed within 24 hours
(On scale of $0-10$, what was the worst pain in the last 12 hours from admission?)

$\bigcirc$ Yes

No

unavailable

(Did the patient have nausea or vomiting in the last 12 hours from admission?)

$\bigcirc$ Yes

No

unavailable

(Rebound tenderness refers to presence of pain that is more intense when the examiner releases pressure than when palpating the abdomen. Guarding refers to spasm of abdominal wall muscles detected on palpation.)

$\bigcirc$ Yes

No

(It refers to disorientation, somnolence, lethargy, stupor, or coma)

$\bigcirc$ Yes

No

unavailable

(Pleural effusions identified on physical exam, chest X-ray or computed tomography (CT) scan within 24 hours from presentation) 


\section{Laboratory Markers}

Are the laboratory markers available from the time of admission?

WBC on admission ( $x, x x x /$ micro-liters)

Hematocrit on admission (\%, with 1 decimal)

BUN on admission $(\mathrm{mg} / \mathrm{dL})$

Creatinine on admission ( $\mathrm{mg} / \mathrm{dL}$, with 1 decimal)

Lipase level on admission

Lipase Upper Limit of Normal

Admission in this questionnaire refers to the time that the patient presented to the initial center and not the transferred center.
Yes

No

(Are the laboratory markers available from the time of admission to the primary center?)

(White blood count measured upon presenting to initial hospital (not the referal hospital in case of transfer))

(Hematocrit measured upon presenting to initial hospital (not the referal hospital in case of transfer))

(Blood urea nitrogen measured upon presenting to initial hospital (not the referal hospital in case of transfer))

(Creatinine measured upon presenting to initial hospital (not the referal hospital in case of transfer))

(Lipase measured upon presenting to initial hospital (not the referal hospital in case of transfer)) 


\section{At 24 hours}

Record ID

\section{Vital Signs}

Temperature at 24 hours (in Celsius, with 1 decimal)

(Highest temperature recording between 12-24 hours from admission)

Heart rate at 24 hours (beats/min)

(Highest heart rate recording between 12-24 hours from admission)

Respiratory rate at 24 hours (breaths/min)

(Highest respiratory rate recording between $12-24$ hours from admission)

\section{Physical Examinations}

Pain at 24 hours

Nausea/vomiting at 24 hours
(On scale of $0-10$, what was the worst pain between 12-24 hours from admission?)

Yes

No

(Did the patient have nausea or vomiting between 12-24 hours from admission?)

\section{Laboratory Markers}

WBC at 24 hours $(x, x x x /$ microliters $)$

Hematocrit at 24 hours (\%, with 1 decimal)

BUN at 24 hours $(\mathrm{mg} / \mathrm{dL})$

Creatinine at 24 hours $(\mathrm{mg} / \mathrm{dL}$, with 1 decimal)

Lipase level at 24 hours

Lipase Upper Limit of Normal
(Highest white blood count measured between 12-24 hours from admission)

(Highest hematocrit measured between 12-24 hours from admission)

(Highest blood urea nitrogen between 12-24 hours from admission)

(Highest creatinine measured between 12-24 hours from admission)

(Lipase measured upon presenting to initial hospital (not the referal hospital in case of transfer)) 


\section{Intravenous Fluid Therapy}

Type of intravenous fluids within first 6 hours of presentation

Other Intravenous Fluids

Amount of normal saline within first 6 hours of presentation (in milliliters)

Amount of lactated ringers within first 6 hours of presentation (in milliliters)

Amount of other intravenous fluids within first 6 hours of presentation (in milliliters)

Type of intravenous fluids within the first 24 hours

Other Intravenous Fluids

Total amount of normal saline within the first 24

hours (in milliliters)

Total amount of lactated ringers within the first 24 hours (in milliliters)

Total amount of other intravenous fluids within the first 24 hours (in milliliters)

\author{
No intravenous fluid \\ $\square$ Normal saline \\ $\square$ Lactated Ringers \\ Other \\ $\square$ Unavailable \\ (Type of intravenous fluids administered within \\ first 6 hours since patient presentation to \\ initial hospital)
}

(Please write the name of the other intravenous fluids which were used within 6 hours of admission)

(How much normal saline was administered within first 6 hours since patient presentation to initial hospital ? Add boluses and continuing drips.)

(How much lactated ringers was administered within first 6 hours since patient presentation to initial hospital ?)

(How much other intravenous fluids was administered within 6 hours since patient presentation to initial hospital ? )

$\square$ Normal saline

$\square$ Lactated Ringers

$\square$ Other

$\square$ Unavailable

(Type of intravenous fluids administered during the first 24 hours from admission including the first 6 hours)

(Please write the name of the other intravenous fluids which were used within 24 hours of admission)

(Includes total amount of normal saline given during the first 24 hours from admission )

(Includes lacted ringers given during the first 24 hours from admission )

(Includes other IVFs given during the first 24 hours from admission ) 


\section{Pain Management}

Narcotics (day 1)

Common Narcotics

Total amount of Morphine (IV, SC) administered during the first 24 hours of admission

Total amount of Morphine (PO) administered during the first 24 hours of admission

Total amount of Fentanyl (IV) administered during the first 24 hours of admission

Total amount of Hydromorphine (PO) administered during the first 24 hours of admission

Total amount of Hydromorphine (IV, SC) administered during the first 24 hours of admission

Total amount of Oxycodone (PO) administered during the first 24 hours of admission

Total amount of Oxymorphine (PO) administered during the first 24 hours of admission

Total amount of Hydrocodone (PO) administered during the first 24 hours of admission

Total amount of Codeine combinations (PO)

administered during the first 24 hours of admission

Total amount of Methadone (PO) administered during the first 24 hours of admission

Total amount of Meperidine (PO) administered during the first 24 hours of admission

Total amount of Meperidine (IV) administered during the first 24 hours of admission

Other types of analgesics $\bigcirc$ Yes

No

unavailable

(Were oral or parenteral narcotics administered?)

Morphine (IV, SC)

$\square$ Morphine (PO)

Fentanyl (IV, SC)

Hydromorphone (PO)

$\square$ Hydromorphone (IV, SC)

$\square$ Oxycodone (PO)

$\square$ Oxymorphine (PO)

$\square$ Hydrocodone (PO)

$\square$ Codeine combinations (PO)

Methadone

$\square$ Meperidine (PO)

Meperidine (SC, IV)

(All doses are in milligrams) 


\section{At 48 hours}

Record ID

\section{Vital Signs}

Temperature at 48 hours (in Celsius, with 1 decimal)

(Highest temperature recording between 36-48 hours from admission)

Heart rate at 48 hours (beats/min)

(Highest heart rate recording between $36-48$ hours from admission)

Respiratory rate at 48 hours (breaths/min)

(Highest respiratory rate recording between $36-48$ hours from admission)

\section{Physical Examinations}

Pain at 48 hours

Nausea/vomiting at 48 hours
(On scale of $0-10$, what was the worst pain between 36-48 hours from admission)

Yes

No

(Did the patient have nausea or vomiting between 36-48 hours from admission?)

\section{Laboratory Markers}

WBC at 48 hours ( $\mathrm{x}, \mathrm{xxx} / \mathrm{microliters)}$

(Highest white blood count measured between 36-48 hours from admission)

Lipase level at 48 hours

(Lipase measured upon presenting to initial hospital (not the referal hospital in case of transfer))

Lipase Upper Limit of Normal

\section{Pain Management}

Narcotics (day 2)

$\bigcirc$ Yes

No

unavailable

(Were oral or parenteral narcotics administered?) 
Common Narcotics (day 2)

Total amount of Morphine (IV, SC) administered within 25-48 hours from admission

Total amount of Morphine (PO) administered within 25-48 hours from admission

Total amount of fentanyl (IV) administered within 25-48 hours from admission

Total amount of Hydromorphone (PO) administered within 25-48 hours from admission

Total amount of Hydromorphone (IV, SC) administered within 25-48 hours from admission

Total amount of Oxycodone (PO) administered within 25-48 hours from admission

Total amount of Oxymorphine (PO) administered within 25-48 hours from admission

Total amount of Hydrocodone (PO) administered within 25-48 hours from admission

Total amount of Codeine combinations (PO)

administered within 25-48 hours from admission

Total amount of Methadone (PO) administered within 25-48 hours from admission

Total amount of Meperidine (PO) administered within 25-48 hours from admission

Total amount of Meperidine (IV) administered within 25-48 hours from admission

Other types of analgesics (day 2) $\square$ Morphine (IV, SC)

$\square$ Morphine (PO)

$\square$ Fentanyl (IV, SC)

$\square$ Hydromorphone (PO)

$\square$ Hydromorphone (IV, SC)

$\square$ Oxycodone (PO)

$\square$ Oxymorphine (PO)

Hydrocodone (PO)

$\square$ Codeine combinations (PO)

$\square$ Methadone

$\square$ Meperidine (PO)

Meperidine (SC, IV)

(All doses are in milligrams) 


\section{At 72 Hours}

Record ID

Is the patient still in the hospital?

Yes

No

\section{Vital Signs}

Temperature at 72 hours (in Celsius, with 1 decimal)

(Highest temperature recording between $60-72$ hours from admission)

Heart rate at 72 hours (beats/min)

(Highest heart rate recording between $60-72$ hours from admission)

Respiratory rate at 72 hours (breaths/min)

(Highest respiratory rate recording between $60-72$ hours from admission)

\section{Physical Examinations}

Pain at 72 hours

(On scale of $0-10$, what was the worst pain between 60-72 hours from admission?)

Nausea/vomiting at 72 hours

$\bigcirc$ Yes

No

(Did the patient have nausea or vomiting between

60-72 hours from admission?)

\section{Laboratory Markers}

WBC at 72 hours ( $x, x x x /$ microliters $)$

(Highest white blood count measured between 60-72 hours from admission)

Lipase level at 72 hours

(Lipase measured upon presenting to initial hospital (not the referal hospital in case of transfer))

Lipase Upper Limit of Normal 


\section{Pain Management}

Narcotics (day 3)

Common Narcotics (day 3)

Total amount of Morphine (IV, SC) administered within 49-72 hours from admission

Total amount of Morphine (PO) administered within 49-72 hours from admission

Total amount of fentanyl (IV) administered within 49-72 hours from admission

Total amount of Hydromorphone (PO) administered within 49-72 hours from admission

Total amount of Hydromorphone (IV, SC) administered within 49-72 hours from admission

Total amount of Oxycodone (PO) administered within 49-72 hours from admission

Total amount of Oxymorphine (PO) administered within 49-72 hours from admission

Total amount of Hydrocodone (PO) administered within 49-72 hours from admission

Total amount of Codeine combinations (PO)

administered within 49-72 hours from admission

Total amount of Methadone (PO) administered within 49-72 hours from admission

Total amount of Meperidine (PO) administered within 49-72 hours from admission

Total amount of Meperidine (IV) administered within 49-72 hours from admission

Other types of analgesics (day 3) $\bigcirc$ Yes

No

unavailable

(Were oral or parenteral narcotics administered?)

Morphine (IV, SC)

$\square$ Morphine (PO)

Fentanyl (IV, SC)

Hydromorphone (PO)

$\square$ Hydromorphone (IV, SC)

$\square$ Oxycodone (PO)

$\square$ Oxymorphine (PO)

$\square$ Hydrocodone (PO)

$\square$ Codeine combinations (PO)

Methadone

$\square$ Meperidine (PO)

$\square$ Meperidine (SC, IV)

(All doses are in milligrams) 


\section{On day 7}

Record ID

\section{Vital Signs}

Is the patient still in the hospital?

$\bigcirc$ Yes

No

Temperature at 7 days (in Celsius,, with 1 decimal)

(Highest temperature recording between 156-168 hours from admission)

Heart rate at 7 days (beats/min)

(Highest heart rate recording between 156-168 hours from admission)

Respiratory rate at 7 days (breaths/min)

(Highest respiratory rate recording between 156-168 hours from admission)

\section{Physical Examinations}

Pain at 7 days

(On scale of $0-10$, what was the worst pain between 156-168 hours from admission?)

Nausea/vomiting at 7 days

$\bigcirc$ Yes

$\bigcirc$ No

(Did the patient have nausea or vomiting between

156-168 hours from admission?)

\section{Laboratory Markers}

WBC at 7 days ( $x, x x x /$ microliters)

(Highest white blood count measured between

156-168 hours from admission)

\section{Pain Management}

Narcotics (day 7)

Yes

No

unavailable

(Were oral or parenteral narcotics administered?) 
Common Narcotics (day 7)

Total amount of Morphine (IV, SC) administered during the day 7 of admission

Total amount of Morphine (PO) administered during 7th days of admission

Total amount of fentanyl (IV) administered during 7th days of admission

Total amount of Hydromorphone (PO) administered during 7th days of admission

Total amount of Hydromorphone (IV, SC) administered during 7th days of admission

Total amount of Oxycodone (PO) administered during 7th days of admission

Total amount of Oxymorphine (PO) administered during 7th days of admission

Total amount of Hydrocodone (PO) administered during 7th days of admission

Total amount of Codeine combinations (PO)

administered during 7th days of admission

Total amount of Methadone (PO) administered during 7th days of admission

Total amount of Meperidine (PO) administered during 7th days of admission

Total amount of Meperidine (IV) administered during 7th days of admission

Other types of analgesics (day 7

$\square$ Morphine (IV, SC)
$\square$ Morphine (PO)
$\square$ Fentanyl (IV, SC)
$\square$ Hydromorphone (PO)
$\square$ Hydromorphone (IV, SC)
$\square$ Oxycodone (PO)
$\square$ Oxymorphine (PO)
$\square$ Hydrocodone (PO)
$\square$ Codeine combinations (PO)
$\square$ Methadone
$\square$ Meperidine (PO)
$\square$ Meperidine (SC, IV)
(All doses are in milligrams)

(All doses are in milligrams) 


\section{At discharge}

Record ID

\section{Laboratory Markers}

Discharge date

$\overline{\text { (Date the patient was discharged.) }}$

SIRS (Systemic inflammatory response syndrome) criteria

\section{SIRS \\ defined by presence of two or more criteria \\ Heart rate $>90$ beats $/ \mathrm{min}$}

Core temperature $<36^{\circ} \mathrm{C}$ or $>38^{\circ} \mathrm{C}$

White blood count $<4000$ or $>12000 / \mathrm{mm} 3$

\section{Respirations $>20 /$ min or PCO2 $<32$ mm Hg13}

SIRS (Systemic inflammatory response syndrome)

Date and time of SIRS onset

SIRS duration

Triglyceride measurement

Date and time of Triglyceride measurement $\bigcirc$ Yes

No

(Did the patient develop positive SIRS during hospitalization?)

Admission

Day 1

Day 2

Day 3

After day 3

(When did the patient develop positive SIRS for the first time?)

Less than 48 hours

More than 48 hours

(How many hours in total did the patient have positive SIRS until resolution or organ failure development?)

Yes

No

(Are serum Triglyceride (TG) levels available within 48 hours of admission?) 
Triglyceride level (mg/dL)

(Highest triglyceride level measured within 48 hours of admission)

\section{Management/Narcotics}

Total days of narcotics administered

(How many days did the patient receive narcotics (oral or intravenous)?)

\section{Management/ICU}

Intensive Care Unit (ICU) admission

$\bigcirc$ Yes

No

(Was the patient admitted to ICU for further care?)

Date and time of intensive care unit (ICU) admission

Death while in Intensive Care Unit (ICU)

Yes

No

Intensive Care Unit (ICU) length of stay (days)

(length of ICU stay in days; when multiple ICU admissions during same hospitalization, record the total length of stay)

\section{Management/Nutrition}

Date and time of initial feeding attempt

Type of initial oral feeding

Initial feeding route

Tolerance of initial feeding attempt
(Initial feeding attempt includes: oral intake, enteral, or parenteral nutrition)

clear liquid

full liquid

$\bigcirc$ soft mechanical

low-fat

regular diet

(Which type of oral diet was tolerated by patient in initial feeding?)

Oral

$\bigcirc$ Enteral nutrition- gastric route [Nasogastric (NG) or Percutaneous endoscopic gastrostomy(PEG)]

$\bigcirc$ Enteral nutrition- enteral route [Nasojejunal (NJ) or Percutaneous endoscopic jejunostomy (PEJ)]

$\bigcirc$ Total parenteral nutrition (TPN)

(Which feeding route was attempted initially after acute pancreatitis onset?)

Yes

No

(Did the patient tolerate the initial feeding attempt (for at least 24 hours) ? ) 
Second feeding attempt

Route of second feeding attempt

Date and time of second feeding attempt

Type of second oral feeding

Tolerance of second feeding

Third feeding attempt

Date and time of third feeding

Route of third feeding attempt

Type of third oral feeding

Tolerance of third feeding

Oral tolerance $\bigcirc$ Yes

No

(Was there second and different feeding attempt?)

Oral

$\bigcirc$ Enteral nutrition- gastric route [Nasogastric (NG) or Percutaneous endoscopic gastrostomy(PEG)]

$\bigcirc$ Enteral nutrition- enteral route [Nasojejunal (NJ) or Percutaneous endoscopic jejunostomy (PEJ)]

$\bigcirc$ Total parenteral nutrition (TPN)

(Which feeding route was used following initial feeding attempt?)

(When did the patient start second different feeding following initial attempt?)

clear liquid

full liquid

soft mechanical

low-fat

$\bigcirc$ regular diet

(Which type of oral diet was tolerated by patient in second feeding?)

Yes

No

(Did the patient tolerate the second feeding attempt (for at least 24 hours)?)

Yes

No

(Was there third different feeding attempt?)

(When did the patient start third different feeding following second attempt?)

no

Oral

$\bigcirc$ Enteral nutrition- gastric route [Nasogastric (NG) or Percutaneous endoscopic gastrostomy(PEG)]

$\bigcirc$ Enteral nutrition- enteral route [Nasojejunal (NJ) or Percutaneous endoscopic jejunostomy (PEJ)]

$\bigcirc$ Total parenteral nutrition (TPN)

(What feeding route was used in third feeding attempt?)

clear liquid

$\bigcirc$ full liquid

$\bigcirc$ soft mechanical

low-fat

$\bigcirc$ regular diet

(Which type of oral diet was tolerated by patient in third feeding?)

Yes

No

(Did the patient tolerate the third feeding attempt (for at least 24 hours)?)

(When did the patient tolerate oral feeding (for at least 24 hours)?) 


\section{Management/Early Intervention}

Early pancreatic intervention

Type of early pancreatic intervention

Mode of early pancreatic intervention

Date of early pancreatic intervention

ERCP during hospitalization

Date of first ERCP

ERCP indication

Cholecystectomy during hospitalization

Date of cholecystectomy $\bigcirc$ Yes

No

(Early intervention on the pancreas or peripancreatic tissues within 2 weeks from presentation (ERCP and cholecystectomy are excluded))

$\bigcirc$ Drainage only

Drainage and debridement

(Early pancreatic intervention can include only drrainage of necroma, or both drainage and necrosectomy (debridement))

Laparotomy

Minimally invasive surgery (laparoscopic, retroperitoneal, etc)

Percutaneous catheter drainage

Endoscopic drainage/debridement

(The date of intervention on pancreas or perpancreatic tissues within 2 weeks from presentation (ERCP and cholecystectomy are excluded ))

$\bigcirc$ Yes

No

(ERCP stands for endoscopic retrograde cholangiopancreatography )

Common bile duct stone

Jaundice without bile duct stone

$\bigcirc$ Pancreatic duct injury

$\bigcirc$ other

$\bigcirc$ Yes

No

\section{Complications during hospitalization}

Organ Failure (choose more than one if indicated)

no

$\square$ cardiovascular (systolic blood pressure $<90 \mathrm{mmHg}$ (not fluid responsive), $\mathrm{pH}<7.3$, or use of inotropes)

$\square$ respiratory ( $\mathrm{PaO} 2 / \mathrm{FiO} 2<300$, or need for intubation)

$\square$ renal [(serum creatinine $>1.8 \mathrm{mg} / \mathrm{dL}$ or $>169$ $\mu \mathrm{mol} / \mathrm{L}$, or need to hemodialysis), if there is no pre-existing renal failure]

(Organ failure based on modified Marshall score)

Date and time of organ failure onset 
System that failed first (choose more than one if indicated)

Cardiovascular failure duration

Respiratory failure duration

Renal failure duration

Total length of Organ Failure

Extrapancreatic Infection

Type of extra-pancreatic infection $\square$ Cardiovascular
$\square$ Pulmonary
$\square$ Renal

Transient ( $<48$ hours)

$\bigcirc$ Persistent ( $>=48$ hours)

(When systolic blood pressure $<90 \mathrm{mmHg}$ not fluid responsive, $\mathrm{pH}<7.3$, or use of inotropes)

Transient $(<48 \mathrm{~h})$

Persistent $(>=48 \mathrm{~h})$

(When $\mathrm{PaO} 2 / \mathrm{FiO} 2<300$, or need to intubation)

Transient $(<48 \mathrm{~h})$

Persistent $(>=48 \mathrm{~h})$

(When serum $\mathrm{Cr}>1.8 \mathrm{mg} / \mathrm{dl}$ or $>169 \mu \mathrm{mol} / \mathrm{l}$, or need to hemodialysis. [If there is no pre-existing renal failure]

(Total days of organ failure)

Yes

No

(This includes extrapancreatic infections that developed during hospitalization)

Respiratory infection

Urinary Tract Infection

Catheter-related bacteremia

Clostridium difficile

Cholangitis

$\square$ other

\section{Early Radiologic Findings}

\section{(Choose the CECT scan closest to 7 days of admission)}

Contrast-enhanced computed tomography (CECT) scan

Yes

No

(Was CECT scan performed during hospitalization or follow-up within 1 month?)

Date of CECT

(In cases of more than 1 CECT scans, choose the one closest to day 7)

CECT findings

Normal Pancreas

Interstitial Edematous Pancreatitis

$\bigcirc$ Pancreatic Necrosis

Extent of pancreatic necrosis

$\bigcirc<30 \%$

. $30 \%-50 \%$

$>50 \%$

(Percent of total pancreas necrosis) 
Peripancreatic Necrosis

Infected Necrosis

Diagnostic method of infected necrosis no

yes

not recorded in our center

(Presence of heterogeneous areas of non-enhancement on CECT scan in the peripancreatic area that contain non-liquefied, ill-defined components, nodular areas of increased peripancreatic fat attenuation with visual density higher than simple fluid and considerably higher than simple stranding)

Yes

No

Culture of surgical specimen

$\bigcirc$ Culture of FNA specimen

Based on imaging

Clinical suspicion only

Other

(How infected necrosis was diagnosed?)

\section{Late Radiologic Findings}

(Choose the CECT scan closest to 1 month of admission)

Walled-off Necrosis

Date of walled-off necrosis

Largest diameter of walled-off necrosis (centimeters)
Yes

$\bigcirc$ No

(Walled-off necrosis defines as encapsulated collection of pancreatic and/or peripancreatic necrosis that has developed a well-defined borders)

(When was the walled-off necrosis identified?)

(How much is the largest diameter of walled-off necrosis (reported in CECT)?)

\section{Severity classification}

Revised Atlanta Classification

mild acute pancreatitis

$\bigcirc$ moderately severe acute pancreatitis

$\bigcirc$ severe acute pancreatitis 


\section{Box 3 Grades of severity}

- Mild acute pancreatitis

- No organ failure

- No local or systemic complications

- Moderately severe acute pancreatitis

- Organ failure that resolves within $48 \mathrm{~h}$ (transient organ failure) and/or

- Local or systemic complications without persistent organ failure

- Severe acute pancreatitis

- Persistent organ failure ( $>48 \mathrm{~h}$ )

-Single organ failure

-Multiple organ failure

Determinant-based Classification

mild acute pancreatitis

moderate acute pancreatitis

severe acute pancreatitis

$\bigcirc$ critical acute pancreatitis

Determinat-based Clasiffication definitions

TABLE 1. Determinant-Based Classification of Acute Pancreatitis Severity

\begin{tabular}{|l|c|c|c|c|}
\hline & $\begin{array}{c}\text { Mild } \\
\text { AP }\end{array}$ & $\begin{array}{c}\text { Moderate } \\
\text { AP }\end{array}$ & $\begin{array}{c}\text { Severe } \\
\text { AP }\end{array}$ & $\begin{array}{c}\text { Critical } \\
\text { AP }\end{array}$ \\
\hline $\begin{array}{c}\text { (Peri)pancreatic } \\
\text { necrosis }\end{array}$ & No & Sterile & Infected & Infected \\
\hline & $A N D$ & $A N D / O R$ & OR & AND \\
\hline & No & Transient & Persistent & Persistent \\
\hline
\end{tabular}




\section{Clinical outcomes}

Mortality in hospital

$\bigcirc$ Yes

No

(Patient death during hospitalization)

Cause of Mortality

related to acute pancreatitis

$\bigcirc$ unrelated to acute pancreatitis

(Did the patient die during hospitalization due to complications of acute pancreatitis which include organ failure or secondary infection?)

Date of death

Total Hospital Length of Stay (days)

(Total hospital length of stay in days. If patient is transferred add length of stay in both the initial and the referral hospital)

\section{Patient Satisfaction}

During this stay at the hospital, how often were you treated with courtesy and respect?

During this stay at the hospital, how often was your pain well controlled?
Never (0-10\%)

Sometimes (10-50\%)

$\bigcirc$ Usually (50-90\%)

Always (90-100\%)

(To be answered before discharge by the patient)

Never $(0-10 \%)$

Sometimes (10-50\%)

$\bigcirc$ Usually (50-90\%)

Always (90-100\%)

(To be answered before discharge by the patient) 


\section{APPRENTICE \\ Acute Pancreatitis Patient Registry To Examine Novel Therapies In Clinical Experiences}

\section{$\underline{\text { Specific Aims }}$}

- Prospective collection of demographic, clinical, laboratory, and radiologic data in acute pancreatitis patients from several centers throughout the world with central storage of de-identified data at the University of Pittsburgh

- Evaluation of the existing risks, predictive scores, and markers of severe disease and allocation of patients in the two recent severity classifications based on their clinical course

- Evaluation of the current management and outcomes of acute pancreatitis around the world.

\section{Background and Significance}

Acute pancreatitis (AP) is an acute inflammatory process of the pancreas with variable clinical course but generally is characterized by sudden onset of upper abdominal pain radiating to the back, nausea, epigastric tenderness and elevation of pancreatic digestive enzymes ( e.g. amylase and lipase) in the serum and urine. Currently, AP is the leading cause of GI related admissions in the US hospitals resulting in high physical and financial burden (Gastroenterology 2012;143:1179-87.e1-3). Most cases are mild and selflimited; however, around $20 \%$ of AP cases result in local or systemic complications associated with high morbidity and mortality that can reach up to 30\% (Gut 2013;62:10211).

Over the last 2 decades there has been increased interest in evaluating clinical severity of patients with AP. This research has led to the revision of disease definitions and severity classification. Examples of commonly used AP classification systems are Revised Atlanta Classification Group (Gut 2013;62:102-11) and the Determinant Based Classification (Ann Surg 2012;256: 875-880) systems. In addition, available clinical scores and markers at predicting the severity of AP are only moderately accurate (Mounzer R. Gastroenterology 2012).

The management of AP is largely based on expert opinions. Further large randomized controlled trials are needed and novel therapeutic approaches are necessary in order to provide foundations for determining best course of treatment/s, symptom management, and develop novel therapeutic approaches. 
Further challenges may be explained by limitations in current studies in which the statistical power is limited because of small patient population and/or because they are conducted in a single center. In order to address these issues, we propose a multi-center, international, collaboration of major AP centers to develop a network of qualified investigators throughout the world and enroll large number of subjects into an online database. The results of this study and development of this database will show the feasibility of developing multicenter, international protocols in AP aiming to identify risks and improve treatment of AP.

\section{Methods:}

This is a multi-center, prospective study, which will aim to recruit and follow hospitalized patients with AP. This study is coordinated by the pancreas group at the University of Pittsburgh Medical Center (UPMC) and supported by the Collaborative Alliance for Pancreatic Education and Research community (CAPER). The study will include adults with confirmed diagnosis of AP admitted to the hospital. Each center's research team will determine patient's eligibility to participate in this research study.

This is an observational study, collecting clinical data in patients with AP. Data collection will include: severity of symptoms, pain, demographics, laboratory markers, radiologic findings, management, hospital course, and outcomes. Our primary outcome variables are presence of persistent organ failure and pancreatic necrosis as those two are the main determinants of severity suggested by the two revised severity classifications (Revised Atlanta Classification and Determinant-Based Classification). Based on those two main outcomes we will evaluate existing risks, predictive scores, and markers of severe disease. Furthermore, we will evaluate current management practices in AP patients around the world. Secondary outcomes that will be studied include need for ICU, need for nutritional support, need for intervention, hospital length of stay and mortality.

De-identified data from each center will be recorded in an online standardized questionnaire through the REDCap website. Research coordinators gather data through both direct interview and patients' clinical records. Those variables, which are required to be collected through patient interview, are labeled by brackets in the questionnaire. Completion of this questionnaire takes, on average, 45 minutes, while patient interviews are usually less than $\mathbf{3 0}$ minutes. The research coordinator and investigators at each center will be provided with a unique password protected username to access REDCap. They will be responsible for verifying patients' eligibility and data entry.

The questionnaire is designed to gather information about patient demographics, pancreatic disease history, family history, alcohol use, current medications, clinical characteristics, diagnostic tests, current therapies, hospital course, interventions and disease classification. Patients will be contacted within 3090 days after discharge from the hospital to complete a follow-up questionnaire. The follow-up questionnaire will mainly focus on recurrent attacks of AP, the need to delay intervention, and the potential development of AP-related complications, i.e. 
diabetes mellitus and exocrine pancreatic insufficiency.

\section{$\underline{\text { Recruitment Procedures: }}$}

Recruitment will be accomplished using the Investigators' and co-investigators' own patient population at each center. Every principal investigator and co-investigator have been selected based on their expertise in AP research. Investigators will correctly diagnose the patients with AP and review the inclusion and exclusion criteria according to protocol. Eligible patients will be approached by study personnel and the study will be explained to them. In the event that the patient is not able to give consent (e.g. intubated and unable to talk) the patient's proxy will complete the consent form. Patients who are interested in participating in the study will be given a detailed approved consent form that explains the study and informs them of the potential risks and benefits associated with participation in the study. After all of the patients questions and concerns are addressed by the study coordinator and/or investigator and the consent form is signed, the research coordinator and/or investigator will conduct the interview. This will occur during the patient's hospital stay. The participant will then be contacted after 30 to 90 days post discharge from hospital.

\section{Power and statistical approach:}

We plan to recruit 5,000 cases in one year. For the evaluation of existing predictive scores, z- statistic will be utilized for sample size calculation since both predictive scores and primary outcomes are dichotomous variables. Continuous data will be evaluated for normality of distribution by the Kolmogorov-Smirnov test (or other). Normally distributed data will be presented as mean values \pm standard deviation (SD), whereas data that are not normally distributed as median values with interquartile range (IQR). Differences between two groups with continuous data will be assessed using the student- $t$ test for normally distributed data and the Mann-Whitney test for non-normal data distributions. Comparisons of three or more groups of data will be made using one-way analysis of variance (ANOVA) and Kruskal-Wallis (non-parametric ANOVA) tests. Discrete data will be compared by the chi-square or chi-square trend test depending on the number of groups. A two-sided p-value of less than 0.05 will be considered statistically significant.

\section{Patient Identification:}

The racial, gender and ethnic characteristics of the proposed subject population reflects the demographics of the approved research center and surrounding communities participating in this study. No exclusion criteria shall be based on race, ethnicity, gender or HIV status.

\section{Inclusion Criteria:}

1. The diagnosis of AP based upon presence of two out of the three following criteria: 
a. Abdominal pain typical to AP

b. Serum amylase or lipase levels more than three times the upper limit of normal

c. Imaging findings suggestive of AP

2. Willingness to participate in the study and ability to sign informed consent by patient or his/her proxy (if unable to speak).

\section{Exclusion criteria:}

1. Age under 18 years

2. Unwilling to provide consent by patient or his/her proxy

3. Presence of pancreatic cancer

4. Presence of chronic pancreatitis

5. Occurrence of AP following a multiple trauma episode

6. Having history of organ transplant

7. Presence of any cancer which required chemotherapy or radiation therapy in the past year.

\section{Risks and Benefits:}

The possibility that the results of the research study will become generally known is rare and occurs in less than $1 \%$ (less than 1 out of 100 people). We developed a process, which is detailed in the Data Safety and Monitoring section, in order to reduce the chances of this from occurring.

There is no direct benefit to the patient for participation in this study. The information obtained from this study may lead to greater knowledge of AP.

\section{Data Safety and Monitoring:}

All the data will be collected and stored prospectively on an online database (REDCap) accessible by study personnel at each center. The data will be de-identified and assigned a study code before storage. REDCap is an established secure online software used to access the study material (e.g. questioners), enter and save the collected data, and communicate with other sites about the latest news regarding the study. The data will be monitored by the data coordinator at the Pittsburgh Coordinating Site. All data and safety issues will be discussed at regularly scheduled DSM meetings with the PI. 2. The data will be de- 
identified by each site and the link of study code to study code to identity will be maintained by each site. No identifying information will be entered into the database.

Every center will have access to their own data. Raw data from all centers will be stored centrally in the REDCap coordinating site at the University of Pittsburgh. The data will be accessible by the analysis and publication committee of APPRENTICE with their members including Dr. Papachristou (PI) and additional principal investigators from other geographical areas. All collaborators will be invited to propose research ideas based on their expertise and experience and will have an opportunity to lead one of the projects. The committee will be in charge of assigning projects to individual investigators and setting a time frame for completion. An experienced statistician at the coordinating or leading center based on resources, will have access to the relevant de-identified data so as to complete the statistical analysis for each project.

\section{Cost and Compensation:}

There are no costs to the participant or the participants insurance for procedures conducted for research purposes only.

There is no compensation to those patients participating in this study.

\section{Qualifications of Investigators:}

PI-Georgios Papachristou, M.D., is an Associate Professor at the Division of Gastroenterology, Hepatology and Nutrition, University of Pittsburgh. Dr. Papachristou has conducted an extensively important researches focused on AP and continues to do research and clinical studies on AP. He has over 100 publications and many federal and foundation grants. ?

David C. Whitcomb, M.D., Ph.D., is a Professor of Medicine in the Division of Gastroenterology and Hepatology, Department of Medicine, Cell Biology and Physiology, and Human Genetics, University of Pittsburgh, and Chief of the Division of Gastroenterology and Hepatology.

Dhiraj Yadav, M.D., is an Associate Professor of Medicine in the Division of Gastroenterology, Hepatology and Nutrition, University of Pittsburgh. He is an expert in epidemiology and alcoholic pancreatitis.?

Amir Gougol, M.D., is a research scholar with the University of Pittsburgh, Department of Medicine, Gastroenterology division.? ?

Efstratios Koutroumpakis, M.D., is a research scholar with the University of Pittsburgh, Department of Medicine, Gastroenterology division.??

Venkata Akshintala, M.D., is a resident of Internal Medicine at UPMC? ? 
Kim Stello is a member of the research staff with the University of Pittsburgh, Department of Medicine, Gastroenterology division.? ? ?

Danielle Dwyer is a member of the research staff with the University of Pittsburgh, Department of Medicine, Gastroenterology division.? ?

Gregory Owens, BA, CCRP is a research coordinator in the Department of Medicine, Division of Gastroenterology, University of Pittsburgh 